\title{
Formalizing Single-assignment Program Verification: an Adaptation-complete Approach
}

\author{
Cláudio Belo Lourenço, Maria João Frade, and \\ Jorge Sousa Pinto \\ HASLab/INESC TEC \& Universidade do Minho, Portugal
}

\begin{abstract}
Deductive verification tools typically rely on the conversion of code to a single-assignment (SA) form. In this paper we formalize program verification based on the translation of While programs annotated with loop invariants into a dynamic single-assignment language with a dedicated iterating construct, and the subsequent generation of compact, indeed linear-size, verification conditions. Soundness and completeness proofs are given for the entire workflow, including the translation of annotated programs to SA form. The formalization is based on a program logic that we show to be adaptation-complete. Although this important property has not, as far as we know, been established for any existing program verification tool, we believe that adaptation-completeness is one of the major motivations for the use of SA form as an intermediate language. Our results here show that indeed this allows for the tools to achieve the maximum degree of adaptation when handling subprograms.
\end{abstract}

\section{Introduction}

In the last years deductive program verification has reached a stage of a certain maturity, to the point that a number of tools are now available allowing users to prove properties of programs written in real-world languages like C, Java, or SPARK 36[1025. Deductive techniques attempt to establish the correctness of a software system with respect to a specification, usually given as a set of contracts expressed in first-order logic. Their precision depends on information provided by the user in the form of annotations, in particular loop invariants.

Three trends have characterized the development of modern program verifiers: first, they employ Satisfiability Modulo Theories (SMT) solvers to check the validity of first-order formulas. The nuclear component is a Verification Conditions Generator (VCGen), that takes as input a program and a specification, and produces a set of first-order proof obligations that are sent to a solver. If all the conditions are valid, then the program is correct. The second trend is that deductive verification tools are usually generic, based on programming languages tailored for verification. Rather than producing from scratch a dedicated verifier, programs of a particular language are translated into the intermediate language of the tool, together with a background encoding of the relevant aspects of that language. Two widely used generic verifiers are Boogie [4] and Why3 [13]. 
Finally, and this is the subject of the present paper, modern tools employ internally a Single-Assignment (SA) representation of the code [11, in which variables may not be assigned after they have been read or written. Not only are SA branching programs easier to encode logically, but its use also solves a fundamental inefficiency issue. Verification conditions generated from standard imperative code may be of exponential size in the size of the programs, which destroys any hope of effectively verifying reasonably-sized programs. However, Flanagan and Saxe 14 have shown that conversion of the code to SA form allows for the generation of quadratic size VCs (a technique that achieves conditions of linear-size with respect to the SA program was later proposed [5]). Other advantages have to do with the fact that intermediate values of computations are never lost: when an instruction like $x:=x+1$ is executed, one variable will store the initial value of $x$, and a new variable will store its new value. Specification languages like ACSL, for ANSI-C [7, often allow the value of a variable at a given program point to be used in assertions. In an SA setting this amounts to simply fetching the adequate "version variable". This also means that continuous invariants (that are not relevant for a loop but may be required after it) are transported automatically, and do not have to be explicitly included.

On the theoretical side, the foundations of program verification have traditionally lied in two different frameworks: Dijkstra's predicate transformers for a guarded commands language 12 and program logics, like Hoare logic [17] and separation logic [26]. Guarded commands have been used as an intermediate language in tools like ESC/Java 22] and more recently the Boogie generic verifier. Many pragmatic aspects of program verification have been addressed and described in this setting, in particular the generation of efficiently provable Verification Conditions (VCs) and the treatment of unstructured programs [14/5]. The program logic tradition on the other hand, which is based on separate operational and axiomatic semantics of programming languages, has allowed for the study of properties like soundness and (relative) completeness of Hoare logic with respect to the standard semantics of a While language [2, an approach that has been extended with the treatment of pointers and aliasing in separation logic.

An important issue is that of modular verification and proof reuse. Ideally, one produces a separate proof of correctness for each occurrence (or call) of a subprogram $C$ inside a program $P$, and then adapts the proved specification of $C$ to different 'local' specifications. A formalism that always allows for this to be done is said to be adaptation-complete [20]; in its original formulation Hoare logic is not adaptation-complete. This is a problem in the presence of recursive procedures, since it leads to incompleteness of the program logic itself, but it is also a problem for the implementation of tools where the correctness of each procedure is proved once and for all with respect to a contract that must be adapted to the local context of each call to it. Our work shows that adaptationcompleteness is a natural property of reasoning in the single-assignment setting.

Contributions. In this paper we formalize a verification technique for While programs annotated with invariants, based on their conversion to an intermediate SA form. Verification tools convert programs to single-assignment form internally 
and profit from this in various ways, in particular to achieve efficiency and to handle subprograms - our technique is a minimal model of such a tool. It relies on (i) a novel notion of single-assignment program that supports loops annotated with invariants; (ii) a notion of translation of While programs annotated with loop invariants (resp. Hoare triples containing such programs) into SA programs (resp. Hoare triples containing SA programs); (iii) a Hoare-style logic for these programs; and (iv) a VCGen generating linear-size verification conditions for Hoare triples containing SA programs. The entire workflow is proved to be sound and complete - in particular, we show how invariants annotated in the initial While program are translated into the intermediate SA form in a way that guarantees the completeness of the approach. This means that if the invariants annotated in the original program are appropriate for showing its correctness, then the verification of the translated SA program will be successful.

An adaptation-complete variant of the logic is also proposed, by adding to the inference system a dedicated consequence rule with a simple side condition. This new consequence rule is restricted to reasoning about triples in which the program does not assign any variable occurring free in the precondition; since the Hoare logic for SA programs propagates preconditions forward in a way that preserves this property, the rule can be applied at any point in a derivation. It provides the highest degree of adaptation, without the need to check any additional complicated conditions or rules, as used to be the case in adaptationcomplete presentations of Hoare logic [2|120].

As an added bonus, this paper can also be seen as bridging a gap between two different theoretical traditions - the guarded commands/predicate transformers setting, where the use of single-assignment form was first introduced for the sake of proof efficiency, and the Hoare logic tradition, that formalizes reasoning with loop invariants based on a standard interpretation of imperative programs.

The paper is organized as follows: Section 2 contains background material. In Section 3 we introduce a language of iterating SA programs: loops are annotated with invariants; they have single-assignment bodies; and a renaming allows for the values of the initial variables to be updated between iterations. We propose a Hoare-style partial correctness program logic for this language in Section 4 its inference system admits only derivations guided by the annotated loop invariants, following a forward-propagation strategy. We also give an algorithm that generates compact conditions (in the sense of Flanagan and Saxe 14]) for a given Hoare triple, and then optimize it to generate linear-sized VCs. The next sections contain our main results. We first consider the verification workflow based on the translation of annotated While programs to the SA language. We identify, in Section 5 , the semantic requirements that are expected from such a translation. The workflow is validated by showing that the generation of VCs from the SA form is sound and complete for the verification of the initial program (a concrete translation is given in the appendix, together with the proof that it meets the requirements). In Section 6 we show how the program logic can be extended with a special consequence rule that makes it adaptation-complete. Finally Section 7 discusses related work and Section 8 concludes the paper. 


\section{Hoare Logic}

We briefly review Hoare logic for While programs. The logic deals with the notion of correctness of a program w.r.t. a specification.

Syntax. We consider a typical While language whose commands $C \in \mathbf{C o m m}$ are defined over a set of variables $x \in$ Var in the following way:

$$
C::=\text { skip }|x:=e| C ; C \mid \text { if } b \text { then } C \text { else } C \mid \text { while } b \text { do } C
$$

We will not fix the language of program expressions $e \in \operatorname{Exp}$ and Boolean expressions $b \in \mathbf{E x p}^{\text {bool }}$, both constructed over variables from Var (a standard instantiation is for Exp to be a language of integer expressions and $\mathbf{E x p}^{\mathbf{b o o l}}$ constructed from comparison operators over Exp, together with Boolean operators). In addition to expressions and commands, we need formulas that express properties of particular states of the program. Program assertions $\phi, \theta, \psi \in$ Assert (preconditions and postconditions in particular) are formulas of a first-order language obtained as an expansion of $\mathbf{E x p}^{\text {bool }}$.

We also require a class of formulas for specifying the behaviour of programs. Specifications are pairs $(\phi, \psi)$, with $\phi, \psi \in$ Assert intended as precondition and postcondition for a program. The precondition is an assertion that is assumed to hold when the program is executed, whereas the postcondition is required to hold when its execution stops. A Hoare triple, written as $\{\phi\} C\{\psi\}$, expresses the fact that the program $C$ conforms to the specification $(\phi, \psi)$.

Semantics. We will consider an interpretation structure $\mathcal{M}=(D, I)$ for the vocabulary describing the concrete syntax of program expressions. This structure provides an interpretation domain $D$ as well as a concrete interpretation of constants and operators, given by $I$. The interpretation of expressions depends on a state, which is a function that maps each variable into its value. We will write $\Sigma=\operatorname{Var} \rightarrow D$ for the set of states (note that this approach extends to a multi-sorted setting by letting $\Sigma$ become a generic function space). For $s \in \Sigma$, $s[x \mapsto a]$ will denote the state that maps $x$ to $a$ and any other variable $y$ to $s(y)$. The interpretation of $e \in \mathbf{E x p}$ will be given by a function $\llbracket e \rrbracket_{\mathcal{M}}: \Sigma \rightarrow D$, and the interpretation of $b \in \mathbf{E x p}^{\text {bool }}$ will be given by $\llbracket b \rrbracket_{\mathcal{M}}: \Sigma \rightarrow\{\mathbf{F}, \mathbf{T}\}$. This reflects our assumption that an expression has a value at every state (evaluation always terminates without error) and that expression evaluation never changes the state (the language is free of side effects). For the interpretation of assertions we take the usual interpretation of first-order formulas, noting two facts: since assertions build on the language of program expressions their interpretation also depends on $\mathcal{M}$ (possibly extended to account for user-defined predicates and functions), and states from $\Sigma$ can be used as variable assignments in the interpretation of assertions. The interpretation of the assertion $\phi \in$ Assert is then given by $\llbracket \phi \rrbracket_{\mathcal{M}}: \Sigma \rightarrow\{\mathbf{F}, \mathbf{T}\}$, and we will write $s=\phi$ as a shorthand for $\llbracket \phi \rrbracket_{\mathcal{M}}(s)=\mathbf{T}$. In the rest of the paper we will omit the $\mathcal{M}$ subscripts for the sake of readability; the interpretation structure will be left implicit. 
1. $\langle$ skip, $s\rangle \sim s$

2. $\langle x:=e, s\rangle \sim s[x \mapsto \llbracket e \rrbracket(s)]$

3. if $\left\langle C_{1}, s\right\rangle \sim s^{\prime}$ and $\left\langle C_{2}, s^{\prime}\right\rangle \sim s^{\prime \prime}$, then $\left\langle C_{1} ; C_{2}, s\right\rangle \sim s^{\prime \prime}$

4. if $\llbracket b \rrbracket(s)=\mathbf{T}$ and $\left\langle C_{t}, s\right\rangle \sim s^{\prime}$, then $\left\langle\right.$ if $b$ then $C_{t}$ else $\left.C_{f}, s\right\rangle \leadsto s^{\prime}$

5. if $\llbracket b \rrbracket(s)=\mathbf{F}$ and $\left\langle C_{f}, s\right\rangle \sim s^{\prime}$, then $\left\langle\right.$ if $b$ then $C_{t}$ else $\left.C_{f}, s\right\rangle \sim s^{\prime}$

6. if $\llbracket b \rrbracket(s)=\mathbf{T},\langle C, s\rangle \sim s^{\prime}$ and $\left\langle\right.$ while $b$ do $\left.C, s^{\prime}\right\rangle \sim s^{\prime \prime}$, then $\langle$ while $b$ do $C, s\rangle \sim s^{\prime \prime}$

7. if $\llbracket b \rrbracket(s)=\mathbf{F}$, then $\langle$ while $b$ do $C, s\rangle \sim s$

Fig. 1: Evaluation semantics for While programs

For commands, we consider a standard operational, natural style semantics, based on a deterministic evaluation relation $\leadsto \subseteq \mathbf{C o m m} \times \Sigma \times \Sigma$ (which again depends on an implicit interpretation of program expressions). We will write $\langle C, s\rangle \sim s^{\prime}$ to denote the fact that if $C$ is executed in the initial state $s$, then its execution terminates, and the final state is $s^{\prime}$. The usual inductive definition of this relation is given in Figure 1 .

The intuitive meaning of the triple $\{\phi\} C\{\psi\}$ is that if the program $C$ is executed in an initial state in which the precondition $\phi$ is true, then either execution of $C$ does not terminate or if it does, the postcondition $\psi$ will be true in the final state. Because termination is not guaranteed, this is called a partial correctness specification. Let us define formally the validity of a Hoare triple.

Definition 1. The Hoare triple $\{\phi\} C\{\psi\}$ is said to be valid, denoted $\mid=\{\phi\} C\{\psi\}$, whenever for all $s, s^{\prime} \in \Sigma$, if $s=\phi$ and $\langle C, s\rangle \sim s^{\prime}$, then $s^{\prime} \models \psi$.

Hoare Calculus. Hoare [17 introduced an inference system for reasoning about Hoare triples, which we will call system $\mathrm{H}$ - see Figure 2 (left). Note that the system contains one rule (conseq) whose application is guarded by first-order conditions. We will consider that reasoning in this system takes place in the context of the complete theory $\operatorname{Th}(\mathcal{M})$ of the implicit structure $\mathcal{M}$, so that when constructing derivations in $\mathrm{H}$ one simply checks, when applying the (conseq) rule, whether the side conditions are elements of $\operatorname{Th}(\mathcal{M})$. We will write $\vdash_{\mathrm{H}}\{\phi\} C\{\psi\}$ to denote the fact that the triple is derivable in this system with $\operatorname{Th}(\mathcal{M})$.

System $\mathrm{H}$ is sound w.r.t. the semantics of Hoare triples; it is also complete as long as the assertion language is sufficiently expressive (a result due to Cook 9] ). One way to ensure this is to force the existence of a strongest postcondition for every command and assertion. Let $C \in \mathbf{C o m m}$ and $\phi \in \mathbf{A s s e r t}$, and denote by $\operatorname{post}(\phi, C)$ the set of states $\left\{s^{\prime} \in \Sigma \mid\langle C, s\rangle \leadsto s^{\prime}\right.$ for some $s \in$ $\Sigma$ such that $\llbracket \phi \rrbracket(s)=\mathbf{T}\}$. In what follows we will assume that the assertion language Assert is expressive with respect to the command language Comm and interpretation structure $\mathcal{M}$, i.e., for every $\phi \in$ Assert and $C \in \mathbf{C o m m}$ there exists $\psi \in$ Assert such that $s \models \psi$ iff $s \in \operatorname{post}(\phi, C)$ for any $s \in \Sigma$. The reader is directed to [2] for details.

Proposition 1 (Soundness of system H). Let $C \in \mathbf{C o m m}$ and $\phi, \psi \in$ Assert. If $\vdash_{\mathbf{H}}\{\phi\} C\{\psi\}$, then $\models\{\phi\} C\{\psi\}$. 


\begin{tabular}{|c|c|c|}
\hline$\overline{\{\phi\} \text { skip }\{\phi\}}$ & (skip) & $\overline{\{\phi\} \operatorname{skip}\{\psi\}}$ if $\phi \rightarrow \psi$ \\
\hline$\overline{\{\psi[e / x]\} x:=e\{\psi\}}$ & (assign) & $\overline{\{\phi\} x:=e\{\psi\}}$ if $\phi \rightarrow \psi[e / x]$ \\
\hline$\{\phi\} C_{1}\{\theta\} \quad\{\theta\} C_{2}\{\psi\}$ & (seq) & $\{\phi\} C_{1}\{\theta\}$ \\
\hline$\{\phi\} C_{1} ; C_{2}\{\psi\}$ & & $\{\phi\} C_{1} ; C_{2}\{\psi\}$ \\
\hline$\{\phi \wedge b\} C_{t}\{\psi\} \quad\{\phi \wedge \neg b\} C_{f}\{\psi\}$ & (if) & $\{\phi \wedge \neg b\} C_{f}\{\psi\}$ \\
\hline$\{\phi\}$ if $b$ then $C_{t}$ else $C_{f}\{\psi\}$ & & $\{\phi\}$ if $b$ then $C_{t}$ else $C_{f}\{\psi\}$ \\
\hline$\{\theta \wedge b\} C\{\theta\}$ & (while) & $\{\theta \wedge b\} C\{\theta\}$ \\
\hline$\overline{\{\theta\} \text { while } b \text { do } C\{\theta \wedge \neg b\}}$ & & $\overline{\{\phi\} \text { while } b \operatorname{do}\{\theta\} C\{\psi\}}$ if $\theta \wedge \neg b \rightarrow \psi$ \\
\hline$\frac{\{\phi\} C\{\psi\}}{\left\{\phi^{\prime}\right\} C\left\{\psi^{\prime}\right\}}$ if $\begin{array}{l}\phi^{\prime} \rightarrow \phi \\
\psi \rightarrow \psi^{\prime}\end{array}$ and & (conseq) & \\
\hline
\end{tabular}

Fig. 2: Systems $\mathrm{H}$ (left) and $\mathrm{Hg}$ (right)

Proposition 2 (Completeness of system H). Let $C \in \mathbf{C o m m}$ and $\phi, \psi \in$ Assert. With Assert expressive in the above sense, if $\models\{\phi\} C\{\psi\}$, then $\vdash_{\mathrm{H}}$ $\{\phi\} C\{\psi\}$.

The sets of variables occurring and assigned in the program $C$ will be given by $\operatorname{Vars}(C)$ and $\operatorname{Asgn}(C)$ respectively; $\mathrm{FV}(\phi)$ denotes the set of free variables occurring in $\phi$ (all are defined in the obvious way). We will write $\phi \# C$ to denote $\operatorname{Asgn}(C) \cap \mathrm{FV}(\phi)=\emptyset$, i.e. $C$ does not assign variables occurring free in $\phi$.

Lemma 1. Let $\phi, \psi \in$ Assert and $C \in \mathbf{C o m m}$, such that $\phi \# C$. If $\vdash_{\mathbf{H}}\{\phi\} C\{\psi\}$, then $\vdash_{\mathbf{H}}\{\phi\} C\{\phi \wedge \psi\}$.

Goal-directed Logic. We introduce a syntactic class AComm of annotated programs, which differs from Comm only in the case of while commands, which are of the form while $b$ do $\{\theta\} C$ where the assertion $\theta$ is a loop invariant annotation (see for instance [15]). Annotations do not affect the operational semantics. Note that for $C \in \mathbf{A C o m m}, \operatorname{Vars}(C)$ includes the free variables of the annotations in $C$. In what follows we will use the auxiliary function $\lfloor\cdot\rfloor:$ AComm $\rightarrow$ Comm that erases all annotations from a program (defined in the obvious way).

In Figure 2 (right) we present system $\mathrm{Hg}$, a goal-directed version of Hoare logic for triples containing annotated programs. This system is intended for mechanical construction of derivations: loop invariants are not invented at this point but taken from the annotations, and there is no ambiguity in the choice of rule to apply, since a consequence rule is not present. The possible derivations of the same triple in $\mathrm{Hg}$ differ only in the intermediate assertions used. The following can be proved by induction on the derivation of $\vdash_{\mathrm{Hg}}\{\phi\} C\{\psi\}$. 


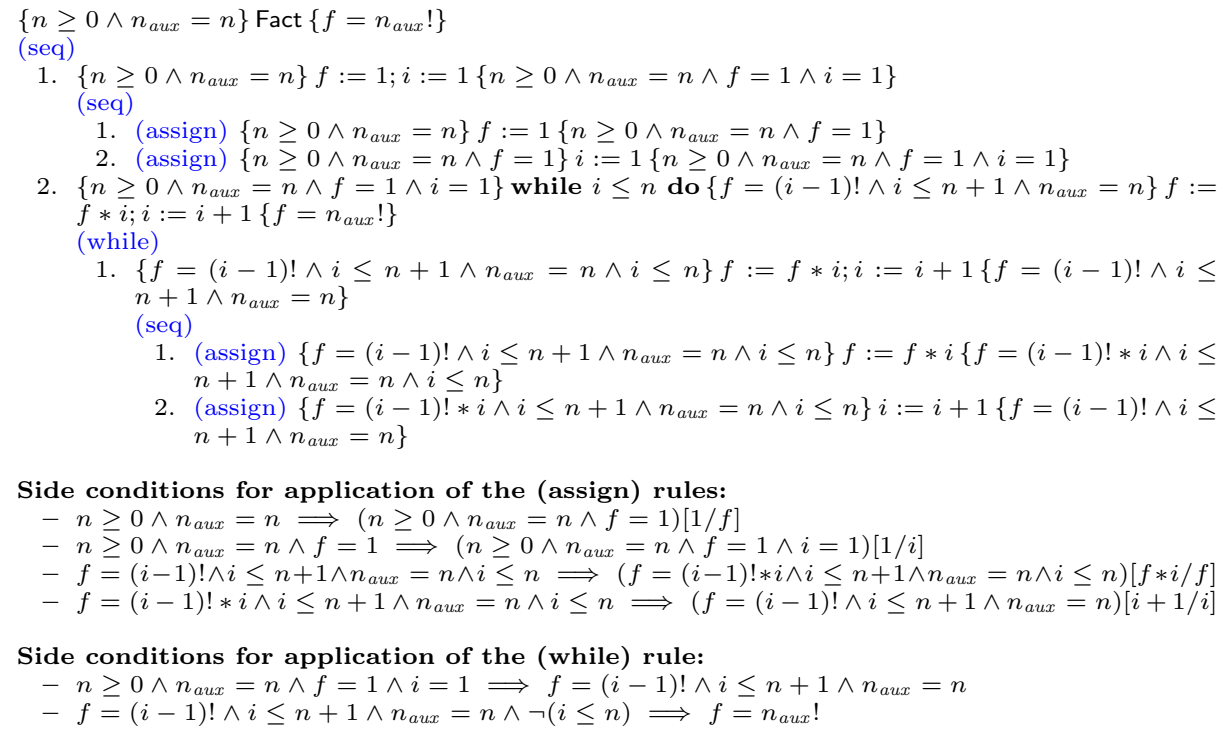

Fig. 3: Example derivation in system $\mathrm{Hg}$

Proposition 3 (Soundness of $\mathrm{Hg}$ ). Let $C \in \mathbf{A C o m m}$ and $\phi, \psi \in$ Assert. If $\vdash_{\mathrm{Hg}}\{\phi\} C\{\psi\}$ then $\vdash_{\mathrm{H}}\{\phi\}\lfloor C\rfloor\{\psi\}$.

The converse implication does not hold, since the annotated invariants may be inadequate for deriving the triple. Instead we need the following definition:

Definition 2. Let $C \in \mathbf{A C o m m}$ and $\phi, \psi \in \mathbf{A s s e r t}$. We say that $C$ is correctlyannotated w.r.t. $(\phi, \psi)$ if $\vdash_{\mathrm{H}}\{\phi\}\lfloor C\rfloor\{\psi\}$ implies $\vdash_{\mathrm{Hg}}\{\phi\} C\{\psi\}$.

The following lemma states the admissibility of the consequence rule in $\mathrm{Hg}$.

Lemma 2. Let $C \in \mathbf{A C o m m}$ and $\phi, \psi, \phi^{\prime}, \psi^{\prime} \in \mathbf{A s s e r t}$ such that $\vdash_{\mathrm{Hg}}\{\phi\} C\{\psi\}$, $=\phi^{\prime} \rightarrow \phi$, and $\models \psi \rightarrow \psi^{\prime}$. Then $\vdash_{\mathrm{Hg}}\left\{\phi^{\prime}\right\} C\left\{\psi^{\prime}\right\}$.

Consider the factorial program shown in Figure 4a. The counter $i$ ranges from 1 to $n$ and the accumulator $f$ contains at each step the factorial of $i-1$. The program is annotated with an appropriate loop invariant; it is easy to show that it is correct with respect to the specification $\left(n \geq 0 \wedge n_{\text {aux }}=n, f=n_{\text {aux }}\right.$ !). We show in Figure 3 a derivation of this triple in system $\mathrm{Hg}$. Note that the axioms $0 !=1$ and $n !=n *(n-1)$ ! are required to prove the side conditions.

It is possible to write an algorithm, known as a verification conditions generator, that simply collects the side conditions of a derivation without actually constructing it. $\mathrm{Hg}$ is agnostic with respect to a strategy for propagating assertions, but the VCGen necessarily imposes one such strategy [15]. 
Exponential Explosion. To understand the exponential explosion problem mentioned in Section 1. consider a program consisting of a sequence of $n$ conditional statements: since each such statement doubles the number of execution paths, the program has $2^{n}$ paths. Consider now the (if) rule of Hoare logic. If one uses a backward propagation strategy, one starts with a given postcondition $\psi$, which will be propagated through both branches of the last conditional, to produce two assertions $\phi_{t}, \phi_{f}$, both of which may contain occurrences of $\psi$. These will be combined in an assertion $\phi$, for instance $\left(b \rightarrow \phi_{t}\right) \wedge\left(\neg b \rightarrow \phi_{f}\right)$, where $\psi$ may occur twice. The (seq) rule will then use $\phi$ as postcondition for the prefix of the program, repeating the process and generating the exponential pattern. A similar exponential pattern may be generated by duplicating variables rather than assertions, in a sequence of assignment statements whose right-hand sides contain multiple occurrences of the same variable. For instance propagating backwards an assertion containing a single occurrence of $z$ through the sequence $y:=x+x ; z:=y+y$ produces a formula containing 4 occurrences of $x$.

Adaptation Incompleteness. Consider a block of code that has been proved correct with respect to a specification. Take for instance the triple $\left\{n \geq 0 \wedge n_{\text {aux }}=\right.$ $n\}$ Fact $\left\{f=n_{\text {aux }}\right.$ ! $\}$. The specification makes use of an auxiliary variable $n_{\text {aux }}$. These variables do not have a special status; they are simply not used as program variables, and can be safely employed for writing specifications relating the pre-state and post-state. According to the above, the program Fact computes the factorial of the initial value of $n$. Now suppose Fact is part of a bigger program $P$, and one would like to establish the validity of the triple $\{n=K\}$ Fact $\{f=K !\}$, with $K$ a positive constant. Adaptation-completeness would mean that one would be able to derive this from the specification of Fact without constructing a dedicated proof - indeed, it should not even be necessary to know the implementation of Fact, since it has already been proved correct. The (conseq) rule of Hoare logic is meant precisely for this, but it cannot be applied here, since both side conditions are clearly not valid. This shows that system $\mathrm{H}$ is not adaptation-complete:

$$
\frac{\left\{n \geq 0 \wedge n_{\text {aux }}=n\right\} \text { Fact }\left\{f=n_{\text {aux }} !\right\}}{\{n=K\} \text { Fact }\{f=K !\}} \quad \text { if } \begin{aligned}
n & =K \rightarrow n \geq 0 \wedge n_{\text {aux }}=n \quad \text { and } \\
f & =n_{\text {aux }} ! \rightarrow f=K !
\end{aligned}
$$

\section{Single-assignment Programs}

Translation of code into Single-Assignment (SA) form has been part of the standard compilation pipeline for decades now; in such a program each variable is assigned at most once. The fragment $x:=10 ; x:=x+10$ could be translated as $x_{1}:=10 ; x_{2}:=x_{1}+10$, using a different "version of $x$ " variable for each assignment. In this paper we will use a dynamic notion of singleassignment (DSA) program [27, in which each variable may occur syntactically as the left-hand side of more than one assignment instruction, as long as it is not assigned more than once in each execution. For instance the fragment 


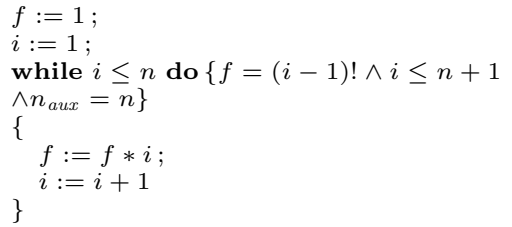

(a) Initial annotated program Fact

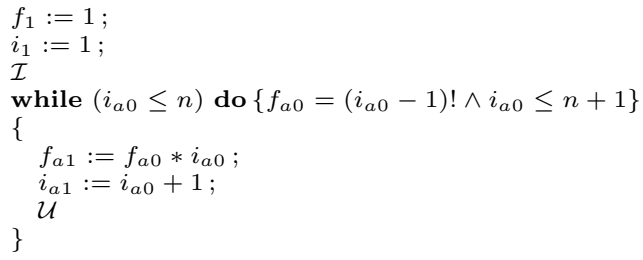

(b) With blocks converted to SA form

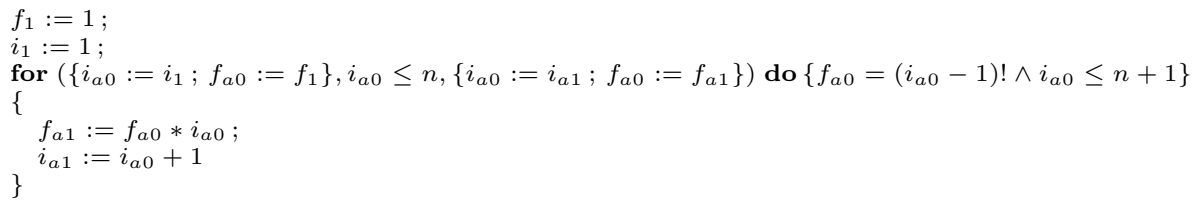

(c) Annotated single-assignment program Fact ${ }^{\text {sa }}$

Fig. 4: Factorial example

if $x>0$ then $x:=x+10$ else skip could be translated into DSA form as if $x_{0}>0$ then $x_{1}:=x_{0}+10$ else $x_{1}:=x_{0}$. Note the else branch cannot be simply skip, since it is necessary to have a single version variable (in this case $x_{1}$ ) representing $x$ when exiting the conditional.

In the context of the guarded commands language, it has been shown 14 that VCs for passive programs (essentially DSA programs without loops, where assignments are replaced with assume commands) can be generated avoiding exponential explosion. However, a single-assignment language of programs with loops, tailored for verification, does not exist. In what follows we will introduce precisely such a language, based on dynamic single-assignment form.

Definition 3. The set $\mathbf{R n m} \subseteq \mathbf{C o m m}$ of renamings consists of all programs of the form $\left\{x_{1}:=y_{1} ; \ldots ; x_{n}:=y_{n}\right\}$ such that all $x_{i}$ and $y_{i}$ are distinct. The empty renaming will be written as skip.

A renaming $\mathcal{R}=\left\{x_{1}:=y_{1} ; \ldots ; x_{n}:=y_{n}\right\}$ represents the finite bijection $\left[x_{1} \mapsto\right.$ $\left.y_{1}, \ldots, x_{n} \mapsto y_{n}\right]$, which we will also denote by $\mathcal{R}$. We will write $\operatorname{dom}(\mathcal{R})$ and $\operatorname{rng}(\mathcal{R})$ to denote the domain and range of $\mathcal{R}$, respectively. Furthermore, $\mathcal{R}(\phi)$ will denote the assertion that results from applying the substitution $\left[y_{1} / x_{1}, \ldots\right.$, $\left.y_{n} / x_{n}\right]$ to $\phi$. Also, for $s \in \Sigma$ we define the state $\mathcal{R}(s)$ as follows: $\mathcal{R}(s)(x)=$ $s(\mathcal{R}(x))$ if $x \in \operatorname{dom}(\mathcal{R})$, and $\mathcal{R}(s)(x)=s(x)$ otherwise.

Lemma 3. Let $\mathcal{R} \in \mathbf{R n m}, \phi, \psi \in$ Assert and $s \in \Sigma$.

1. $\langle\mathcal{R}, s\rangle \sim \mathcal{R}(s)$

2. $\llbracket \mathcal{R}(\phi) \rrbracket(s)=\llbracket \phi \rrbracket(\mathcal{R}(s))$

3. $=\{\phi\} \mathcal{R}\{\psi\} \quad$ iff $\quad \models \phi \rightarrow \mathcal{R}(\psi)$. 
Proof. 1. By inspection on the evaluation relation. 2. By induction on the interpretation assertions. 3. Follows from 1 and 2.

In a strict sense it is not possible to write iterating programs in DSA form. So what we propose here is a syntactically controlled violation of the singleassignment constraints that allows for structured reasoning. Loop bodies are still SA blocks, but two renamings, responsible for propagating the values inside, outside and between iterations, are free of single-assignment restrictions.

Definition 4. Let $\mathbf{A C o m m}{ }^{\mathrm{SA}}$ be the class of annotated single-assignment programs. Its abstract syntax is defined by

$$
C::=\text { skip }|x:=e| C ; C \mid \text { if } b \text { then } C \text { else } C \mid \text { for }(\mathcal{I}, b, \mathcal{U}) \operatorname{do}\{\theta\} C
$$

where:

- skip $\in \mathbf{A C o m m}^{\mathrm{SA}}$

$-x:=e \in \mathbf{A C o m m}^{\mathrm{SA}}$ if $x \notin \operatorname{Vars}(e)$

- $C_{1} ; C_{2} \in \mathbf{A} \mathbf{C o m m}^{\mathrm{SA}}$ if $C_{1}, C_{2} \in \mathbf{A} \mathbf{C o m m}^{\mathrm{SA}}$ and $\operatorname{Vars}\left(C_{1}\right) \cap \operatorname{Asgn}\left(C_{2}\right)=\emptyset$

- if $b$ then $C_{t}$ else $C_{f} \in \mathbf{A C o m m}^{\mathrm{SA}}$ if $C_{t}, C_{f} \in \mathbf{A C o m m}^{\mathrm{SA}}$ and $\operatorname{Vars}(b) \cap\left(\operatorname{Asgn}\left(C_{t}\right) \cup \operatorname{Asgn}\left(C_{f}\right)\right)=\emptyset$

- for $(\mathcal{I}, b, \mathcal{U})$ do $\{\theta\} C \in \mathbf{A C o m m}^{\mathrm{SA}}$ if $C \in \mathbf{A C o m m}^{\mathrm{SA}}, \mathcal{I}, \mathcal{U} \in \mathbf{R n m}, \operatorname{Asgn}(\mathcal{I})=$ $\operatorname{Asgn}(\mathcal{U}), \operatorname{rng}(\mathcal{U}) \subseteq \operatorname{Asgn}(C)$, and $(\operatorname{Vars}(\mathcal{I}) \cup \operatorname{Vars}(b) \cup \mathrm{FV}(\theta)) \cap \operatorname{Asgn}(C)=\emptyset$

and $V$ ars and Asgn are extended to the for command as follows:

- $\operatorname{Vars}($ for $(\mathcal{I}, b, \mathcal{U})$ do $\{\theta\} C)=\operatorname{Vars}(\mathcal{I}) \cup \operatorname{Vars}(b) \cup \mathrm{FV}(\theta) \cup \operatorname{Vars}(C)$

- $\operatorname{Asgn}($ for $(\mathcal{I}, b, \mathcal{U}) \operatorname{do}\{\theta\} C)=\operatorname{Asgn}(\mathcal{I}) \cup \operatorname{Asgn}(C)$

Definition 4 is straightforward except in the case of loops. The initialization code $\mathcal{I}$ contains a renaming that runs exactly once, even if no iterations take place. On the other hand the code in $\mathcal{U}$ is executed after every iteration. This ensures that the variables in $\operatorname{dom}(\mathcal{U})$ (equal to $\operatorname{dom}(\mathcal{I})$ ) always contain the appropriate output values at the beginning of each iteration and when the loop terminates. Note that the definition of $\phi \# C$ extends to annotated programs as expected.

In Figure $4 \mathrm{~b}$ we show again the factorial program, where we have converted the blocks to SA form (the variables occurring in the loop are signalled with an ' $a$ ' subscript for clarity, but any other fresh variables would do). The initial version variables of the loop body $f_{a 0}$ and $i_{a 0}$ are the ones used in the Boolean expression, which is evaluated at the beginning of each iteration. They are also used in the invariant annotation. We have placed in the code the required renamings $\mathcal{I}$ and $\mathcal{U}$, and it is straightforward to instantiate them. $\mathcal{I}$ should be defined as $i_{a 0}:=i_{1} ; f_{a 0}:=f_{1}$, and $\mathcal{U}$ as $i_{a 0}:=i_{a 1} ; f_{a 0}:=f_{a 1}$. The initial version variables can be used after the loop to access the value of the counter and accumulator; so a specification for this program can be written as $\left(n \geq 0 \wedge n_{a u x}=n, f_{a 0}=n_{a u x}\right.$ !). It is now immediate to write the program with a for command encapsulating the structure of the loop, in accordance with Definition 4. This is shown in Figure $4 \mathrm{c}$. Incidentally, note that the invariant does not contain the 'continuous' part $n_{\text {aux }}=n$ of the initial code, since it becomes unnecessary in the SA version.

The function $\mathcal{W}: \mathbf{A C o m m}^{\mathrm{SA}} \rightarrow \mathbf{A C o m m}$ translates SA programs back to (annotated) While programs in the obvious way: $\mathcal{W}($ for $(\mathcal{I}, b, \mathcal{U}) \operatorname{do}\{\theta\} C)=$ $\mathcal{I}$; while $b \operatorname{do}\{\theta\}\{\mathcal{W}(C) ; \mathcal{U}\}$. Otherwise the function is defined as expected. 


$$
\begin{aligned}
& \text { (skip) } \overline{\{\phi\} \text { skip }\{\phi \wedge \top\}} \\
& \text { (assign) } \\
& \text { (seq) } \quad \frac{\{\phi\} C_{1}\left\{\phi \wedge \psi_{1}\right\} \quad\left\{\phi \wedge \psi_{1}\right\} C_{2}\left\{\phi \wedge \psi_{1} \wedge \psi_{2}\right\}}{\{\phi\} C_{1} ; C_{2}\left\{\phi \wedge \psi_{1} \wedge \psi_{2}\right\}} \\
& \text { (if) } \quad \frac{\{\phi \wedge b\} C_{t}\left\{\phi \wedge b \wedge \psi_{t}\right\} \quad\{\phi \wedge \neg b\} C_{f}\left\{\phi \wedge \neg b \wedge \psi_{f}\right\}}{\{\phi\} \text { if } b \text { then } C_{t} \text { else } C_{f}\left\{\phi \wedge\left(\left(b \wedge \psi_{t}\right) \vee\left(\neg b \wedge \psi_{f}\right)\right)\right\}} \\
& \frac{\{\theta \wedge b\} C\{\theta \wedge b \wedge \psi\}}{\{\phi\} \text { for }(\mathcal{I}, b, \mathcal{U}) \text { do }\{\theta\} C\{\phi \wedge \theta \wedge \neg b\}} \text { if } \begin{array}{l}
\phi \rightarrow \mathcal{I}(\theta) \text { and } \\
\theta \wedge b \wedge \psi \rightarrow \mathcal{U}(\theta)
\end{array}
\end{aligned}
$$

Fig. 5: Inference system for annotated SA triples - System Hsa

\section{Logic and Verification Conditions for SA Programs}

We propose in Figure 5 an inference system for Hoare triples containing annotated SA programs. Hsa is goal-directed like system $\mathrm{Hg}$ but it incorporates a strategy, based on forward propagation (reminiscent of strongest postcondition computations). It is proved sound with respect to system $\mathrm{H}$, and complete with respect to $\mathrm{Hg}$. Note that $\mathrm{Hsa}$ derives triples of the form $\{\phi\} C\{\phi \wedge \psi\}$, where the program does not interfere with the truth of the precondition. For this reason we restrict our results to triples satisfying the $\phi \# C$ condition (SA translations will generate triples of this kind only).

Lemma 4. Let $C \in \mathbf{A C o m m}^{\mathrm{SA}}$ and $\phi, \psi \in$ Assert such that $\phi \# C, \vdash_{\mathrm{Hsa}}$ $\{\phi\} C\{\psi\}$. Then (i) $\mathrm{FV}(\psi) \subseteq \mathrm{FV}(\phi) \cup \operatorname{Vars}(C)$ and (ii) all triples $\{\alpha\} C^{\prime}\{\beta\}$ occurring in this derivation satisfy $\alpha \# C^{\prime}$.

Proof. Both are proved by induction on the structure of the derivation of $\vdash_{\mathrm{Hsa}}$ $\{\phi\} C\{\psi\}$.

Proposition 4 (Soundness of system Hsa). Let $C \in \mathbf{A C o m m}^{\mathrm{SA}}$ and $\phi$, $\psi^{\prime} \in$ Assert such that $\phi \# C$. If $\vdash_{\text {Hsa }}\{\phi\} C\left\{\phi \wedge \psi^{\prime}\right\}$, then $\vdash_{\mathrm{H}}\{\phi\}\lfloor\mathcal{W}(C)\rfloor\left\{\phi \wedge \psi^{\prime}\right\}$.

Proof. By induction on the derivation of $\vdash_{\text {Hsa }}\{\phi\} C\left\{\phi \wedge \psi^{\prime}\right\}$, using Lemma 4 and induction hypotheses. We show the interesting case, where the last rule applied is (for). Assume the last step is

$$
\frac{\{\theta \wedge b\} C\{\theta \wedge b \wedge \psi\}}{\{\phi\} \text { for }(\mathcal{I}, b, \mathcal{U}) \operatorname{do}\{\theta\} C\{\phi \wedge \theta \wedge \neg b\}} \quad \text { with } \begin{aligned}
& \phi \rightarrow \mathcal{I}(\theta) \text { and } \\
& \theta \wedge b \wedge \psi \rightarrow \mathcal{U}(\theta)
\end{aligned}
$$

By Lemma 4, we have that $(\theta \wedge b) \# C$. So, by induction hypothesis, we have $\vdash_{\mathrm{H}}\{\theta \wedge b\}[\mathcal{W}(C)\rfloor\{\theta \wedge b \wedge \psi\}$. From the validity of the side conditions, by 
Lemma 3 and completeness of $\mathrm{H}$, we have $\vdash_{\mathrm{H}}\{\theta \wedge b \wedge \psi\} \mathcal{U}\{\theta\}$ and $\vdash_{\mathrm{H}}\{\phi\} \mathcal{I}\{\theta\}$. Now applying sequentially the rules (seq), (while) and again (seq), we get $\vdash_{H}$ $\{\phi\} \mathcal{I}$; while $b$ do $\{\lfloor\mathcal{W}(C)\rfloor ; \mathcal{U}\}\{\theta \wedge \neg b\}$. Hence, by definition of $\mathcal{W}$ and Lemma 1 , we have $\vdash_{\mathrm{H}}\{\phi\}\lfloor\mathcal{W}($ for $(\mathcal{I}, b, \mathcal{U}) \operatorname{do}\{\theta\} C)\rfloor\{\phi \wedge \theta \wedge \neg b\}$.

Proposition 5 (Completeness of system Hsa). Let $C \in \mathbf{A C o m m}^{\mathrm{SA}}$ and $\phi$, $\psi \in$ Assert such that $\phi \# C$ and $\vdash_{\mathrm{Hg}}\{\phi\} \mathcal{W}(C)\{\psi\}$. Then $\vdash_{\text {Hsa }}\{\phi\} C\left\{\phi \wedge \psi^{\prime}\right\}$ for some $\psi^{\prime} \in$ Assert such that $=\phi \wedge \psi^{\prime} \rightarrow \psi$.

Proof. By induction on the structure of $C$. Assume $\phi \# C$ and $\vdash_{\mathrm{Hg}}\{\phi\} \mathcal{W}(C)\{\psi\}$.

- Case $C \equiv x:=e$, we must have $\models \phi \rightarrow \psi[e / x]$. Since $x \notin(\mathrm{FV}(e) \cup \mathrm{FV}(\phi))$, it follows that $\models \phi \wedge x=e \rightarrow \psi$. As $\vdash_{\text {Hsa }}\{\phi\} x:=e\{\phi \wedge x=e\}$ we are done.

- Case $C \equiv C_{1} ; C_{2}$, we must have for some $\gamma \in$ Assert $\vdash_{\mathrm{Hg}}\{\phi\} \mathcal{W}\left(C_{1}\right)\{\gamma\}$ and $\vdash_{\mathrm{Hg}}\{\gamma\} \mathcal{W}\left(C_{2}\right)\{\psi\}$. Since $\phi \# C_{1} ; C_{2}$ we have $\phi \# C_{1}$. Hence by induction hypothesis we have $\vdash_{\text {Hsa }}\{\phi\} C_{1}\left\{\phi \wedge \gamma^{\prime}\right\}$ for some $\gamma^{\prime} \in$ Assert such that $=\phi \wedge \gamma^{\prime} \rightarrow \gamma$. Therefore, by Lemma 2, $\vdash_{\mathrm{Hg}}\left\{\phi \wedge \gamma^{\prime}\right\} \mathcal{W}\left(C_{2}\right)\{\psi\}$. From Lemma 4 we have that $\mathrm{FV}\left(\phi \wedge \gamma^{\prime}\right) \subseteq \mathrm{FV}(\phi) \cup \operatorname{Vars}\left(C_{1}\right)$, and thus $\left(\phi \wedge \gamma^{\prime}\right) \# C_{2}$. Hence by induction hypothesis $\vdash_{\text {Hsa }}\left\{\phi \wedge \gamma^{\prime}\right\} C_{2}\left\{\phi \wedge \gamma^{\prime} \wedge \psi^{\prime}\right\}$ for some $\psi^{\prime} \in$ Assert such that $\models \phi \wedge \gamma^{\prime} \wedge \psi^{\prime} \rightarrow \psi$. Applying rule (seq) we then get $\vdash_{\text {Hsa }}\{\phi\} C_{1} ; C_{2}\left\{\phi \wedge \gamma^{\prime} \wedge \psi^{\prime}\right\}$.

- Case $C \equiv$ for $(\mathcal{I}, b, \mathcal{U})$ do $\{\theta\} C_{t}$, we must have, for some $\gamma \in$ Assert, that $\vdash_{\mathrm{Hg}}\{\phi\} \mathcal{I}\{\theta\}, \vdash_{\mathrm{Hg}}\{\theta \wedge b\} \mathcal{W}\left(C_{t}\right)\{\gamma\}, \vdash_{\mathrm{Hg}}\{\gamma\} \mathcal{U}\{\theta\}$, and $=\theta \wedge \neg b \rightarrow \psi$. We have that $(\theta \wedge b) \# C_{t}$, so it follows by induction hypothesis that $\vdash_{\text {Hsa }}$ $\{\theta \wedge b\} C_{t}\left\{\theta \wedge b \wedge \gamma^{\prime}\right\}$, for some $\gamma^{\prime} \in$ Assert and $=\theta \wedge b \wedge \gamma^{\prime} \rightarrow \gamma$. Therefore, by Lemma 2 . $\vdash_{\mathrm{Hg}}\left\{\theta \wedge b \wedge \gamma^{\prime}\right\} \mathcal{U}\{\theta\}$. Since $\mathrm{Hg}$ is sound, by Lemma 3 , it follows that $=\phi \rightarrow \mathcal{I}(\theta)$ and $=\theta \wedge b \wedge \gamma^{\prime} \rightarrow \mathcal{U}(\theta)$, which allow us to apply rule (for) and get the conclusion $\vdash_{\text {Hsa }}\{\phi\}$ for $(\mathcal{I}, b, \mathcal{U}) \operatorname{do}\{\theta\} C\{\phi \wedge \theta \wedge \neg b\}$.

The remaining cases are routine.

All the rules of system Hsa propagate the precondition $\phi$ forward. Note that in the (for) rule this happens even though $\phi$ is not implied by the annotated loop invariant. Observe also how in this same rule we reason structurally about the body of the loop (an SA piece of code), with the renamings applied to the invariant in the side conditions.

Figure 6 shows an example of a Hsa derivation, where Fact $^{\text {sa }}$ is the factorial single-assignment program of Figure $4 \mathrm{c}$. The assertion $n \geq 0 \wedge n_{\text {aux }}=n$ is used as precondition. Note that the application of the (for) rule introduces two side conditions, which are both valid. The derivation generates a unique postcondition for the program, with the given precondition. Other valid triples with the same precondition may be obtained by weakening this postcondition, following Proposition 5. For the triple $\left\{n \geq 0 \wedge n_{a u x}=n\right\}$ Fact $\left\{f_{a 0}=n_{a u x} !\right\}$ we would check the validity of:

$n \geq 0 \wedge n_{\text {aux }}=n \wedge f_{1}=1 \wedge i_{1}=1 \wedge f_{a 0}=\left(i_{a 0}-1\right) ! \wedge i_{a 0} \leq n+1 \wedge \neg\left(i_{a 0} \leq n\right) \rightarrow f_{a 0}=n_{\text {aux }}$ !

A set of verification conditions for a triple $\{\phi\} C\{\psi\}$ can be obtained from a candidate derivation of a triple of the form $\{\phi\} C\left\{\phi \wedge \psi^{\prime}\right\}$ in system Hsa. The VCs 


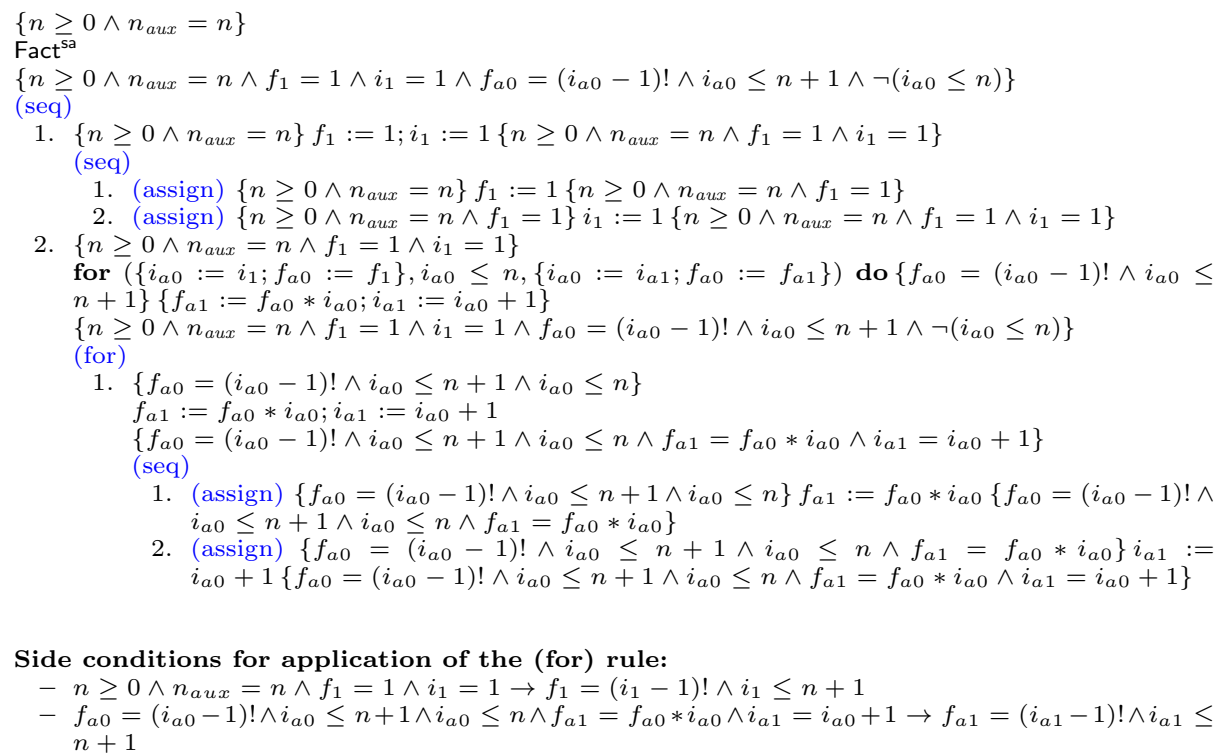

Fig. 6: Example derivation in system Hsa

are the side conditions introduced by the (for) rule, together with $\phi \wedge \psi^{\prime} \rightarrow \psi$ : the triple is valid if and only if all these VCs are valid. It is possible to calculate the VCs and the formula $\psi^{\prime}$ without explicitly constructing the derivation. The following function does precisely this.

Definition 5 (Verification Conditions Generator). The VCGen function VC : Assert $\times \mathbf{A C o m m}^{\mathrm{SA}} \rightarrow$ Assert $\times \mathcal{P}($ Assert $)$ is defined as follows:

$$
\begin{aligned}
\operatorname{VC}(\phi, \text { skip })= & (\top, \emptyset) \\
\operatorname{VC}(\phi, x:=e)= & (x=e, \emptyset) \\
\operatorname{VC}\left(\phi, C_{1} ; C_{2}\right)= & \left(\psi_{1} \wedge \psi_{2}, \Gamma_{1} \cup \Gamma_{2}\right) \\
& \text { where }\left(\psi_{1}, \Gamma_{1}\right)=\operatorname{VC}\left(\phi, C_{1}\right) \text { and } \\
& \left(\psi_{2}, \Gamma_{2}\right)=\operatorname{VC}\left(\phi \wedge \psi_{1}, C_{2}\right) \\
\operatorname{VC}\left(\phi, \text { if } b \text { then } C_{t} \text { else } C_{f}\right)= & \left(\left(b \wedge \psi_{t}\right) \vee\left(\neg b \wedge \psi_{f}\right), \Gamma_{t} \cup \Gamma_{f}\right) \\
& \text { where }\left(\psi_{t}, \Gamma_{t}\right)=\operatorname{VC}\left(\phi \wedge b, C_{t}\right) \text { and } \\
& \left(\psi_{f}, \Gamma_{f}\right)=\operatorname{VC}\left(\phi \wedge \neg b, C_{f}\right) \\
\operatorname{VC}(\phi, \text { for }(\mathcal{I}, b, \mathcal{U}) \operatorname{do}\{\theta\} C)= & (\theta \wedge \neg b, \Gamma \cup\{\phi \rightarrow \mathcal{I}(\theta), \theta \wedge b \wedge \psi \rightarrow \mathcal{U}(\theta)\}) \\
& \text { where }(\psi, \Gamma)=\operatorname{VC}(\theta \wedge b, C)
\end{aligned}
$$

Let $\left(\psi^{\prime}, \Gamma\right)=\operatorname{VC}(\phi, C)$. The verification conditions of $C$ with the precondition $\phi$ are given by the set $\Gamma$, and the formula $\psi^{\prime}$ approximates (since it relies on loop invariants) a logical encoding of the program; it is clear from the definition that $\psi^{\prime}$ does not depend on the formula $\phi$. The VCs of a Hoare triple $\{\phi\} C\{\psi\}$ are then given by $\Gamma \cup\left\{\phi \wedge \psi^{\prime} \rightarrow \psi\right\}$.

Proposition 6. Let $C \in \mathbf{A C o m m}^{\mathrm{SA}}, \phi, \psi^{\prime}, \psi^{\prime \prime} \in$ Assert and $\Gamma \subseteq$ Assert, such that $\left(\psi^{\prime}, \Gamma\right)=\mathrm{VC}(\phi, C)$. Then: 
1. If $\models \Gamma$, then $\vdash_{\text {Hsa }}\{\phi\} C\left\{\phi \wedge \psi^{\prime}\right\}$.

2. If $\vdash_{\text {Hsa }}\{\phi\} C\left\{\phi \wedge \psi^{\prime \prime}\right\}$, then $\models \Gamma$ and $\psi^{\prime \prime} \equiv \psi^{\prime}$.

Proof. 1. By induction on the structure of $C$. 2. By induction on the derivation of $\vdash_{\text {Hsa }}\{\phi\} C\left\{\phi \wedge \psi^{\prime \prime}\right\}$.

The reader may check that for our factorial example we have $\operatorname{VC}(n \geq 0 \wedge$ $n_{\text {aux }}=n$, Fact $)=\left(f_{1}=1 \wedge i_{1}=1 \wedge f_{a 0}=\left(i_{a 0}-1\right) ! \wedge i_{a 0} \leq n+1 \wedge \neg\left(i_{a 0} \leq n\right),\{n \geq\right.$ $0 \wedge n_{\text {aux }}=n \wedge f_{1}=1 \wedge i_{1}=1 \rightarrow f_{1}=\left(i_{1}-1\right) ! \wedge i_{1} \leq n+1, f_{a 0}=\left(i_{a 0}-1\right) ! \wedge i_{a 0} \leq$ $\left.\left.n+1 \wedge i_{a 0} \leq n \wedge f_{a 1}=f_{a 0} * i_{a 0} \wedge i_{a 1}=i_{a 0}+1 \rightarrow f_{a 1}=\left(i_{a 1}-1\right) ! \wedge i_{a 1} \leq n+1\right\}\right)$, in accordance with the derivation of Figure 6 .

Consider the calculation of $\mathrm{VC}\left(\phi,\left\{\right.\right.$ if $b$ then $C_{t}$ else $\left.\left.C_{f}\right\} ; C_{2}\right)$. The recursive call on $C_{2}$ will be $\operatorname{VC}\left(\phi \wedge\left(\left(b \wedge \psi_{t}\right) \vee\left(\neg b \wedge \psi_{f}\right)\right), C_{2}\right)$, where $\psi_{t}, \psi_{f}$ do not depend on $\phi$. The resulting VCs avoid the exponential pattern described in Section 2 , since a single copy of the precondition $\phi$ is propagated to $C_{2}$. In fact the size of the $\mathrm{VCs}$ is quadratic on the size of the program. It is clear from the $\operatorname{VC}\left(\phi, C_{1} ; C_{2}\right)$ clause of the definition that the propagated precondition $\phi$ is duplicated, with one copy used to generate VCs for $C_{1}$, and another propagated to $C_{2}$ together with the encoding of $C_{1}$. Now observe that each loop in the program generates two VCs, one corresponding to the initialization of the invariant $(\phi \rightarrow \mathcal{I}(\theta))$, and another to its preservation. The size of loop preservation VCs depends only on the size of the loop's body, but initialization conditions contain an encoding of the prefix of the program leading to the loop (propagated in the $\phi$ parameter), so they have size linear in the size of that prefix. The worst case occurs for a program consisting in a sequence of $n$ loops: the $i^{\text {th }}$ loop will generate an initialization $\mathrm{VC}$ of size $\mathcal{O}(i)$, so the total size of the VCs is $\mathcal{O}\left(n^{2}\right)$.

This VCGen can in fact be simplified, in a way that potentially decreases the size of the VCs. We have seen that the propagation of assertions (using the $\phi$ parameter) is a potential source of formula duplication, but in fact the $\phi$ parameter can be eliminated. For this, the algorithm must now return a triple $(\psi, \gamma, \Gamma)$ containing, in addition to an encoding $\psi$ of the program and a set $\Gamma$ of VCs, the VC that is currently being constructed (whereas the conditions in $\Gamma$ have already been fully generated, inside inner loops of the current block). The simplified VCGen highlights the fundamental fact that VC generation for SA programs is not a matter of directed 'propagation' of assertions, either forward or backward: it suffices to perform a single program traversal collecting pieces of information along the way, and conveniently structuring them:

$$
\begin{aligned}
& \mathrm{VC}_{\mathrm{L}} \text { (skip) }=(\top, \top, \emptyset) \\
& \operatorname{VC}_{\mathrm{L}}(x:=e)=(x=e, \top, \emptyset) \\
& \mathrm{VC}_{\mathrm{L}}\left(C_{1} ; C_{2}\right)=\left(\psi_{1} \wedge \psi_{2}, \gamma_{1} \wedge\left(\psi_{1} \rightarrow \gamma_{2}\right), \Gamma_{1} \cup \Gamma_{2}\right) \\
& \text { where }\left(\psi_{1}, \gamma_{1}, \Gamma_{1}\right)=\mathrm{VC}_{\mathrm{L}}\left(C_{1}\right) \text { and }\left(\psi_{2}, \gamma_{2}, \Gamma_{2}\right)=\mathrm{VC}_{\mathrm{L}}\left(C_{2}\right) \\
& \mathrm{VC}_{\mathrm{L}}\left(\text { if } b \text { then } C_{t} \text { else } C_{f}\right)=\left(\left(b \wedge \psi_{t}\right) \vee\left(\neg b \wedge \psi_{f}\right),\left(b \rightarrow \gamma_{t}\right) \wedge\left(\neg b \rightarrow \gamma_{f}\right), \Gamma_{t} \cup \Gamma_{f}\right) \\
& \text { where }\left(\psi_{t}, \gamma_{t}, \Gamma_{t}\right)=\mathrm{VC}_{\mathrm{L}}\left(C_{t}\right) \text { and }\left(\psi_{f}, \gamma_{f}, \Gamma_{f}\right)=\operatorname{VC}_{\mathrm{L}}\left(C_{f}\right) \\
& \operatorname{VC}_{\mathrm{L}}(\text { for }(\mathcal{I}, b, \mathcal{U}) \operatorname{do}\{\theta\} C)=(\theta \wedge \neg b, \mathcal{I}(\theta),\{\theta \wedge b \rightarrow \gamma \wedge(\psi \rightarrow \mathcal{U}(\theta))\} \cup \Gamma) \\
& \text { where }(\psi, \gamma, \Gamma)=\operatorname{VC}_{\mathrm{L}}(C)
\end{aligned}
$$


Let $\left(\psi_{l}^{\prime}, \gamma_{l}, \Gamma_{l}\right)=\mathrm{VC}_{\mathrm{L}}(C)$ and $\left(\psi^{\prime}, \Gamma\right)=\mathrm{VC}(\phi, C)$. Clearly $\psi_{l}^{\prime}$ and $\psi^{\prime}$ are the same, and it can be proved by induction that $\bigwedge \Gamma \Leftrightarrow \wedge \Gamma_{l} \wedge\left(\phi \rightarrow \gamma_{l}\right)$. The VCs of a Hoare triple $\{\phi\} C\{\psi\}$ are then given by $\Gamma_{l} \cup\left\{\phi \rightarrow \gamma_{l} \wedge\left(\psi_{l}^{\prime} \rightarrow \psi\right)\right\}$.

With respect to VC size, note that this VCGen joins the initialization VCs of all the top-level loops in each sequence in a single condition. Instead of replicating prefixes of the program for each $\mathrm{VC}$, a single formula is generated, that will be valid only when all initialization conditions hold. For left-associative sequences the size of this formula may still be quadratic, since the sequence clause duplicates the program formula $\psi_{1}$ of $C_{1}$; however, if sequences are represented in a right-associative way (a reasonable assumption for an intermediate language), the size of the resulting VCs is linear in the size of $C$ in the worst-case.

\section{Program Verification Using Intermediate SA Form}

We will now put up a framework for the verification of annotated While programs, based on their translation to single-assignment form and the subsequent generation of compact verification conditions from this intermediate code.

The translation into SA form will operate at the level of Hoare triples, rather than of isolated annotated programs. Such a translation must of course abide by the syntactic restrictions of $\mathbf{A} \mathbf{C o m m}^{\mathrm{SA}}$ (as ilustrated by the factorial example), with additional requirements of a semantic nature. In particular, the translation will annotate the SA program with loop invariants (produced from those contained in the original program), and $\mathrm{Hg}$-derivability guided by these annotations must be preserved. On the other hand, the translation must be sound: it will not translate invalid triples into valid ones. These requirements are expressed by translating back to While programs.

Definition 6 (SA translation). A function $\mathcal{T}$ : Assert $\times \mathbf{A C o m m} \times \mathbf{A s s e r t} \rightarrow$ Assert $\times \mathbf{A C o m m}^{\mathrm{SA}} \times \mathbf{A s s e r t}$ is said to be a single-assignment translation if when $\mathcal{T}(\phi, C, \psi)=\left(\phi^{\prime}, C^{\prime}, \psi^{\prime}\right)$ we have $\phi^{\prime} \# C^{\prime}$, and both the following hold:

1. If $=\left\{\phi^{\prime}\right\}\left\lfloor\mathcal{W}\left(C^{\prime}\right)\right\rfloor\left\{\psi^{\prime}\right\}$, then $=\{\phi\}\lfloor C\rfloor\{\psi\}$.

2. If $\vdash_{\mathrm{Hg}}\{\phi\} C\{\psi\}$, then $\vdash_{\mathrm{Hg}}\left\{\phi^{\prime}\right\} \mathcal{W}\left(C^{\prime}\right)\left\{\psi^{\prime}\right\}$.

The following results establish that translating annotated programs to an intermediate SA form before generating VCs results in a sound and complete technique for deductive verification.

Proposition 7 (Soundness of verification technique). Let $C \in \mathbf{A C o m m}$, $C^{\prime} \in \mathbf{A C o m m}^{\mathrm{SA}}, \phi, \phi^{\prime}, \psi, \psi^{\prime}, \gamma \in \mathbf{A s s e r t}$ and $\Gamma \subseteq$ Assert, such that $\left(\phi^{\prime}, C^{\prime}, \psi^{\prime}\right)=$ $\mathcal{T}(\phi, C, \psi)$ for some $S A$ translation $\mathcal{T}$, and $(\gamma, \Gamma)=\operatorname{VC}\left(\phi^{\prime}, C^{\prime}\right)$.

If $\models \Gamma, \phi^{\prime} \wedge \gamma \rightarrow \psi^{\prime}$ then $\models\{\phi\}\lfloor C\rfloor\{\psi\}$.

Proof. From Proposition 6(1) we have $\vdash_{\text {Hsa }}\left\{\phi^{\prime}\right\} C^{\prime}\left\{\phi^{\prime} \wedge \gamma\right\}$ and from Definition 6 we have $\phi^{\prime} \# C^{\prime}$. Thus Proposition 4 applies yielding $\vdash_{\mathrm{H}}\left\{\phi^{\prime}\right\}\left\lfloor\mathcal{W}\left(C^{\prime}\right)\right\rfloor\left\{\phi^{\prime} \wedge\right.$ $\gamma$ \}. From soundness of $\mathbf{H}$, and because $\models \phi^{\prime} \wedge \gamma \rightarrow \psi^{\prime}$, it follows that $=$ $\left\{\phi^{\prime}\right\}\left\lfloor\mathcal{W}\left(C^{\prime}\right)\right\rfloor\left\{\psi^{\prime}\right\}$. Finally, by Definition 6, we have $=\{\phi\}\lfloor C\rfloor\{\psi\}$. 
Proposition 8 (Completeness of verification technique). Let $C \in \mathbf{A C o m m}$, $C^{\prime} \in \mathbf{A C o m m}^{\mathrm{SA}}, \phi, \phi^{\prime}, \psi, \psi^{\prime}, \gamma \in \mathbf{A s s e r t}$ and $\Gamma \subseteq$ Assert such that $\left(\phi^{\prime}, C^{\prime}, \psi^{\prime}\right)=$ $\mathcal{T}(\phi, C, \psi)$ for some $S A$ translation $\mathcal{T}$, and $(\gamma, \Gamma)=\mathrm{VC}\left(\phi^{\prime}, C^{\prime}\right)$. If $\models\{\phi\}\lfloor C\rfloor\{\psi\}$ and $C$ is correctly-annotated w.r.t. $(\phi, \psi)$, then $\models \Gamma, \phi^{\prime} \wedge \gamma \rightarrow \psi^{\prime}$.

Proof. First note that by completeness of system $\mathrm{H}$ we have $\vdash_{\mathrm{H}}\{\phi\}\lfloor C\rfloor\{\psi\}$. By Definitions 2 and 6 it follows that $\vdash_{\mathrm{Hg}}\{\phi\} C\{\psi\}$ and $\vdash_{\mathrm{Hg}}\left\{\phi^{\prime}\right\} \mathcal{W}\left(C^{\prime}\right)\left\{\psi^{\prime}\right\}$. The latter definition implies that $\phi^{\prime} \# C^{\prime}$, and by Proposition $5 \vdash_{\text {Hsa }}\left\{\phi^{\prime}\right\} C^{\prime}\left\{\phi^{\prime} \wedge \psi^{\prime \prime}\right\}$ for some $\psi^{\prime \prime} \in$ Assert such that $=\phi^{\prime} \wedge \psi^{\prime \prime} \rightarrow \psi^{\prime}$. Proposition 6(2) then gives us $\models \Gamma$ and $\psi^{\prime \prime} \equiv \gamma$, which concludes the proof.

An example of a detailed translation can be found in Appendix A, together with the proof that it complies to Definition 6 .

\section{Adaptation Completeness of SA Program Logic}

Let $(\phi, \psi)$ and $\left(\phi^{\prime}, \psi^{\prime}\right)$ be specifications, and assume that $(\phi, \psi)$ is satisfiable (there exists some program that is correct w.r.t. it). Suppose now that $C$ is a program such that if the Hoare triple $\{\phi\} C\{\psi\}$ is valid then so is $\left\{\phi^{\prime}\right\} C\left\{\psi^{\prime}\right\}$. An inference system for Hoare logic is said to be adaptation-complete if whenever this happens, then $\left\{\phi^{\prime}\right\} C\left\{\psi^{\prime}\right\}$ is derivable in that system from the triple $\{\phi\} C\{\psi\}$.

Adaptation is closely linked to the existence of a consequence rule that dictates when a triple is derivable in one step from another triple containing the same program. Adaptation is by design entirely absent from goal-directed systems like $\mathrm{Hg}$ or $\mathrm{Hsa}$, which have no consequence rule. System $\mathrm{H}$ is capable of adaptation, but not in a complete way. An example of this was already seen at the end of Section 2, involving the use of auxiliary variables. For an even simpler example of how adaptation fails in system $\mathrm{H}$, consider the triple $\{x>0\} P\{y=x\}$ where $x$ is now a program variable, used outside $P$, but not assigned in $P$. Again let $K$ be some positive constant. Clearly if the triple is valid then so is $\{x=K\} P\{y=K\}$, since the value of $x$ is preserved. However, attempting to apply the consequence rule would yield the following, where the first side condition is valid, but the second is invalid

$$
\frac{\{x>0\} P\{y=x\}}{\{x=K\} P\{y=K\}} \quad \text { if } \begin{aligned}
& x=K \rightarrow x>0 \text { and } \\
& y=x \rightarrow y=K
\end{aligned}
$$

The problem of adaptation was raised by the study of complete extensions of Hoare logic for reasoning about recursive procedures. The initial proposal by Hoare [18 was to derive a triple concerning a procedure call by assuming that same triple as a hypothesis when reasoning about the procedure's body:

$$
\frac{\{\phi\} \text { call } \mathbf{p}\{\psi\} \vdash\{\phi\} \operatorname{bod} \mathbf{y}(\mathbf{p})\{\psi\}}{\{\phi\} \text { call } \mathbf{p}\{\psi\}}
$$

(assuming that an identity axiom is present, and system $\mathrm{H}$ rules are lifted to work with sequents). It was soon discovered that, in the presence of auxiliary 
variables in the procedure's specification, the resulting system turned out to be incomplete, and the reason for this was the failure to handle the adaptation of the procedure's specification to the local context of the recursive call.

One solution for this problem was to introduce additional structural rules [1, but Kleymann [20] has shown that the adaptation problem is orthogonal to the handling of recursive procedures: if the base system is made adaptation-complete, then Hoare's rule for recursive procedure calls is sufficient to achieve a Cookcomplete system, with no need for further structural rules.

Kleymann obtains an adaptation-complete inference system for Hoare logic by proposing the following consequence rule, whose side condition is a meta-level formula with quantification over states/variable assignments:

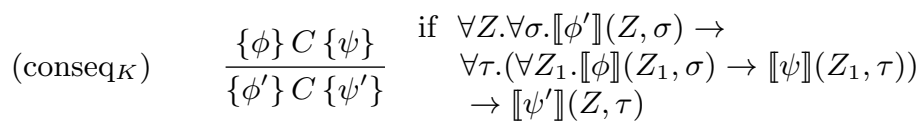

$\llbracket \phi^{\prime} \rrbracket(Z, \sigma)$ denotes the truth value of $\phi^{\prime}$ in the state $(Z, \sigma)$, partitioned between auxiliary $(Z)$ and program $(\sigma)$ variables. As it is, (conseq $\left.{ }_{K}\right)$ cannot be handled directly by an SMT solver, since the condition is not a first-order formula.

We will show that reasoning with single-assignment programs is advantageous from the point of view of adaptation: our Hsa system will be made adaptationcomplete by adding a rule with a simple syntactic side condition. We start with a result showing that the side condition of a consequence rule that always leads to adaptation-completeness, in general terms, turns out to be the result of stripping away the states and quantifiers in the side condition of $\left(\operatorname{conseq}_{K}\right)$ above.

Lemma 5. Let $\phi, \phi^{\prime}, \psi, \psi^{\prime} \in$ Assert. If there exists at least one program $C_{0} \in$ Comm such that $\models\{\phi\} C_{0}\{\psi\}$, and for arbitrary $C$ one has that $\models\{\phi\} C\{\psi\}$ implies $\models\left\{\phi^{\prime}\right\} C\left\{\psi^{\prime}\right\}$, then it must be the case that $\models \phi^{\prime} \rightarrow(\phi \rightarrow \psi) \rightarrow \psi^{\prime}$.

Proof. We assume $\not \forall \phi^{\prime} \rightarrow(\phi \rightarrow \psi) \rightarrow \psi^{\prime}$, i.e. there exists a state $s_{0}$ such that $s_{0} \models \phi^{\prime}, s_{0} \models \phi \rightarrow \psi$, and $s_{0} \not \models \psi^{\prime}$. To show that in this context $\models\left\{\phi^{\prime}\right\} C\left\{\psi^{\prime}\right\}$ does not follow from $=\{\phi\} C\{\psi\}$ for arbitrary $C$, we construct a particular program $C_{1}$ with the following behavior: $\left\langle C_{1}, s_{0}\right\rangle \sim s_{0}$ and for $s \neq s_{0},\left\langle C_{1}, s\right\rangle \sim s^{\prime}$ whenever $\left\langle C_{0}, s\right\rangle \leadsto s^{\prime}$. To see that $\{\phi\} C_{1}\{\psi\}$ is a valid triple, observe that if $s_{0} \models \phi$ and $C_{1}$ is executed in state $s_{0}$ we will have $s_{0} \models \psi$ since $s_{0} \models \phi \rightarrow \psi$, and for other executions we note that $=\{\phi\} C_{0}\{\psi\}$. The triple $\left\{\phi^{\prime}\right\} C_{1}\left\{\psi^{\prime}\right\}$ is however not valid, since $s_{0} \models \phi^{\prime}$, but $\left\langle C_{1}, s_{0}\right\rangle \leadsto s_{0}$ and $s_{0} \not \models \psi^{\prime}$.

The problem is that a consequence rule with side condition $\phi^{\prime} \rightarrow(\phi \rightarrow \psi) \rightarrow \psi^{\prime}$ would not be sound. But it is sound for triples satisfying the simple syntactic restriction that free variables of the precondition are not assigned in the program.

Lemma 6. Let $C \in \mathbf{C o m m}$ and $\phi \in$ Assert. If $\phi \# C$ and $\langle C, s\rangle \sim s^{\prime}$, then $\llbracket \phi \rrbracket(s)=\llbracket \phi \rrbracket\left(s^{\prime}\right)$.

Proof. Since $\phi \# C, s(x)=s^{\prime}(x)$ for every $x \in \mathrm{FV}(\phi)$. Hence, $\llbracket \phi \rrbracket(s)=\llbracket \phi \rrbracket\left(s^{\prime}\right)$. 
Lemma 7. Let $C \in \mathbf{C o m m}$ and $\phi, \phi^{\prime}, \psi, \psi^{\prime} \in$ Assert, such that $\phi \# C$ and $\phi^{\prime} \# C$. If $\models\{\phi\} C\{\psi\}$ and $=\phi^{\prime} \rightarrow(\phi \rightarrow \psi) \rightarrow \psi^{\prime}$, then $=\left\{\phi^{\prime}\right\} C\left\{\psi^{\prime}\right\}$.

Proof. Assume $s=\phi^{\prime}$ and $\langle C, s\rangle \sim s^{\prime}$. Since $\phi^{\prime} \# C$, by Lemma 6, we get $s^{\prime} \models \phi^{\prime}$. We also have $s^{\prime} \models \phi \rightarrow \psi$ because, if $s^{\prime}=\phi$, then $s=\phi$ (by Lemma 6) since $\phi \# C)$ so, as $\models\{\phi\} C\{\psi\}$, we get $s^{\prime} \models \psi$. Now, $s^{\prime} \models \psi^{\prime}$ follows directly from $=\phi^{\prime} \rightarrow(\phi \rightarrow \psi) \rightarrow \psi^{\prime}, s^{\prime} \models \phi^{\prime}$ and $s^{\prime} \models \phi \rightarrow \psi$.

Recall from Lemma 4 that Hsa derivations consist entirely of triples $\{\phi\} C\{\psi\}$ satisfying the $\phi \# C$ condition, which means that an adaptation rule with the side condition given above can be naturally incorporated in the system. We must however be careful to ensure that the new rule preserves Lemma 4 in particular, the postcondition $\psi^{\prime}$ should not contain free occurrences of variables not occurring either in the program or free in the precondition $\phi^{\prime}$. The following result will allow us to eliminate these free occurrences.

Lemma 8. Let $C \in \mathbf{C o m m}, \phi, \psi \in$ Assert and $x \in$ Var, such that $x \notin$ $\mathrm{FV}(\phi) \cup \operatorname{Vars}(C)$. If $\models\{\phi\} C\{\psi\}$ then $\models\{\phi\} C\{\forall x . \psi\}$.

Proof. Assume $s \models \phi$ and $\langle C, s\rangle \sim s^{\prime}$. As $x \notin \mathrm{FV}(\phi) \cup \operatorname{Vars}(C)$, for every $a \in D$, $s[x \mapsto a] \models \phi$ and $\langle C, s[x \mapsto a]\rangle \sim s^{\prime}[x \mapsto a]$. Since $\models\{\phi\} C\{\psi\}$, it follows that $s^{\prime}[x \mapsto a] \models \psi$. Hence, $s^{\prime} \models \forall x . \psi$.

Let $\mathrm{Hsa}^{+}$be the inference system consisting of all the rules of system Hsa together with the following rule:

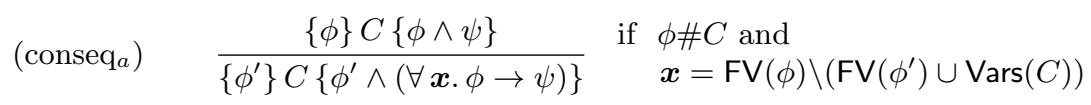

Recall that Hsa is a forward propagation system, so the rule will be applied when we reach $C$ with the propagated precondition $\phi^{\prime}$ (in which case Lemma 4 ensures that $\phi^{\prime} \# C$ holds). The rule will produce a postcondition not directly by propagating $\phi^{\prime}$ through the structure of $C$, but instead by adapting the triple $\{\phi\} C\{\phi \wedge \psi\}$. The conditions $\phi$ and $\psi$ may well contain occurrences of variables not occurring either in $C$ or free in $\phi^{\prime}$, but the quantification ensures that Lemma 4 remains valid in system $\mathrm{Hsa}^{+}$. Note that the lemma guarantees that $\mathrm{FV}(\psi) \subseteq \mathrm{FV}(\phi) \cup \operatorname{Vars}(C)$, so $\mathrm{FV}(\psi)$ does not need to be included in $\boldsymbol{x}$.

System $\mathrm{Hsa}^{+}$is not goal-directed, but it is still a forward-propagation system (the postcondition is the strongest allowed by Lemma 7 ).

Proposition 9 (Soundness of $\mathrm{Hsa}^{+}$). Let $C \in \mathbf{A C o m m}^{\mathrm{SA}}$ and $\phi, \psi^{\prime} \in$ Assert such that $\phi \# C$ and $\vdash_{\mathrm{Hsa}^{+}}\{\phi\} C\left\{\phi \wedge \psi^{\prime}\right\}$. Then $\vdash_{\mathrm{H}}\{\phi\}\lfloor\mathcal{W}(C)\rfloor\left\{\phi \wedge \psi^{\prime}\right\}$.

Proof. The proof, by induction on the structure of the derivation of $\vdash_{\mathrm{Hsa}^{+}}$ $\{\phi\} C\left\{\phi \wedge \psi^{\prime}\right\}$, extends the proof of Proposition 4 with the (conseq ${ }_{a}$ ) rule case. Assume the last step is

$$
\frac{\left\{\phi_{1}\right\} C\left\{\phi_{1} \wedge \psi_{1}\right\}}{\{\phi\} C\left\{\phi \wedge\left(\forall \boldsymbol{x} . \phi_{1} \rightarrow \psi_{1}\right)\right\}} \quad \text { with } \quad \phi_{1} \# C \text { and } \boldsymbol{x}=\mathrm{FV}\left(\phi_{1}\right) \backslash(\mathrm{FV}(\phi) \cup \operatorname{Vars}(C))
$$


By induction hypothesis we have $\vdash_{\mathrm{H}}\left\{\phi_{1}\right\}\lfloor\mathcal{W}(C)\rfloor\left\{\phi_{1} \wedge \psi_{1}\right\}$ and since $\mathrm{H}$ is sound it follows that $\models\left\{\phi_{1}\right\}\lfloor\mathcal{W}(C)\rfloor\left\{\phi_{1} \wedge \psi_{1}\right\}$. As $\models \phi \rightarrow\left(\phi_{1} \rightarrow \phi_{1} \wedge \psi_{1}\right) \rightarrow$ $\phi \wedge\left(\phi_{1} \rightarrow \psi_{1}\right)$, we get $\models\{\phi\}\lfloor\mathcal{W}(C)\rfloor\left\{\phi \wedge\left(\phi_{1} \rightarrow \psi_{1}\right)\right\}$ by Lemma 7 . Now note that $\boldsymbol{x} \cap(\mathrm{FV}(\phi) \cup \operatorname{Vars}(C))=\emptyset$, thus Lemma 8 can be applied, and it follows that $=\{\phi\}\lfloor\mathcal{W}(C)\rfloor\left\{\phi \wedge\left(\forall \boldsymbol{x} . \phi_{1} \rightarrow \psi_{1}\right)\right\}$. Finally, by completeness of $\mathrm{H}$ we obtain $\vdash_{\mathrm{H}}\{\phi\}\lfloor\mathcal{W}(C)\rfloor\left\{\phi \wedge\left(\forall \boldsymbol{x} . \phi_{1} \rightarrow \psi_{1}\right)\right\}$.

The system is obviously complete in the same sense as Hsa, since it extends it. But unlike $\mathrm{Hsa}$ it is also adaptation-complete.

Proposition 10 (Adaptation completeness of $\mathrm{Hsa}^{+}$).

Let $C \in \mathbf{A C o m m}^{\mathrm{SA}}$ and $\phi, \phi^{\prime}, \psi, \psi^{\prime} \in$ Assert such that $\phi \# C,(\phi, \psi)$ is satisfiable, and $=\{\phi\}\lfloor\mathcal{W}(C)\rfloor\{\psi\}$ implies $\models\left\{\phi^{\prime}\right\}\lfloor\mathcal{W}(C)\rfloor\left\{\psi^{\prime}\right\}$.

If $\vdash_{\mathrm{Hsa}^{+}}\{\phi\} C\{\phi \wedge \gamma\}$ for some $\gamma \in$ Assert such that $=\phi \wedge \gamma \rightarrow \psi$, then $\left\{\phi^{\prime}\right\} C\left\{\phi^{\prime} \wedge(\forall \boldsymbol{x} . \phi \rightarrow \gamma)\right\}$ with $\boldsymbol{x}=\mathrm{FV}(\phi) \backslash\left(\mathrm{FV}\left(\phi^{\prime}\right) \cup \operatorname{Vars}(C)\right)$ can be derived from that triple in system $\mathrm{Hsa}^{+}$, and $\models \phi^{\prime} \wedge(\forall \boldsymbol{x} . \phi \rightarrow \gamma) \rightarrow \psi^{\prime}$.

Proof. From $\vdash_{\mathrm{Hsa}^{+}}\{\phi\} C\{\phi \wedge \gamma\}$ we can apply the ( conseq $\left._{a}\right)$ rule to produce $\vdash_{\mathrm{Hsa}^{+}}\left\{\phi^{\prime}\right\} C\left\{\phi^{\prime} \wedge(\forall \boldsymbol{x} . \phi \rightarrow \gamma)\right\}$ with $\boldsymbol{x}=\mathrm{FV}(\phi) \backslash\left(\mathrm{FV}\left(\phi^{\prime}\right) \cup \operatorname{Vars}(C)\right)$, since $\phi \# C$. So it just remains to prove the validity of the formula $\phi^{\prime} \wedge(\forall \boldsymbol{x} . \phi \rightarrow \gamma) \rightarrow \psi^{\prime}$.

From $=\phi \wedge \gamma \rightarrow \psi$ it follows that $=(\phi \rightarrow \gamma) \rightarrow(\phi \rightarrow \psi)$, and so we also have $\models(\forall \boldsymbol{x} . \phi \rightarrow \gamma) \rightarrow(\forall \boldsymbol{x} . \phi \rightarrow \psi)$. Consequently $\models \phi^{\prime} \wedge(\forall \boldsymbol{x} . \phi \rightarrow \gamma) \rightarrow$ $\phi^{\prime} \wedge(\forall \boldsymbol{x} . \phi \rightarrow \psi)$, and thus $\phi^{\prime} \wedge(\forall \boldsymbol{x} . \phi \rightarrow \gamma) \models \phi^{\prime} \wedge(\forall \boldsymbol{x} . \phi \rightarrow \psi)$. On the other hand, as $\boldsymbol{x} \cap \mathrm{FV}\left(\phi^{\prime}\right)=\emptyset$, we have $\phi^{\prime} \wedge(\forall \boldsymbol{x} . \phi \rightarrow \psi) \models \phi^{\prime} \wedge(\phi \rightarrow \psi)$. Now, since $=\{\phi\}\lfloor\mathcal{W}(C)\rfloor\{\psi\}$ implies $=\left\{\phi^{\prime}\right\}\lfloor\mathcal{W}(C)\rfloor\left\{\psi^{\prime}\right\}$, it follows by Lemma 5 that $=\phi^{\prime} \rightarrow(\phi \rightarrow \psi) \rightarrow \psi^{\prime}$, and hence we get $\phi^{\prime} \wedge(\forall \boldsymbol{x} . \phi \rightarrow \gamma) \models \psi^{\prime}$. Now we can conclude that $\models \phi^{\prime} \wedge(\forall \boldsymbol{x} . \phi \rightarrow \gamma) \rightarrow \psi^{\prime}$.

Consider again the example introduced at the end of Section 2. Let $K$ be a positive constant; the Hoare triple $\{n=K\} \operatorname{Fact}^{\text {sa }}\left\{f_{a 0}=K !\right\}$ can now be derived from $\left\{n \geq 0 \wedge n_{\text {aux }}=n\right\} \operatorname{Fact}^{\mathrm{sa}}\left\{n \geq 0 \wedge n_{\text {aux }}=n \wedge f_{a 0}=n_{\text {aux }} !\right\}$ :

$$
\frac{\left\{n \geq 0 \wedge n_{\text {aux }}=n\right\} \operatorname{Fact}^{\text {sa }}\left\{n \geq 0 \wedge n_{\text {aux }}=n \wedge f_{a 0}=n_{\text {aux }} !\right\}}{\{n=K\} \operatorname{Fact}^{\text {sa }}\left\{n=K \wedge\left(\forall n_{\text {aux }} . n \geq 0 \wedge n_{\text {aux }}=n \rightarrow f_{a 0}=n_{\text {aux }} !\right)\right\}}
$$

since $\models n=K \wedge\left(\forall n_{\text {aux }} . n \geq 0 \wedge n_{\text {aux }}=n \rightarrow f_{a 0}=n_{\text {aux }} !\right) \rightarrow f_{a 0}=K$ !. As to the example at the beginning of the present section, consider the following derivation (recall that $x$ is not assigned in $P$ ):

$$
\frac{\{x>0\} P\{x>0 \wedge y=x\}}{\{x=K\} P\{x=K \wedge(x>0 \rightarrow y=x)\}}
$$

This proves $\{x=K\} P\{y=K\}$ since $=x=K \wedge(x>0 \rightarrow y=x) \rightarrow y=K$.

\section{Related Work}

The original notion of Static Single-Assignment (SSA) form [11] limits the syntactic occurrence of each variable as L-value of a single assignment instruction. 
A construct called " $\Phi$-function" is used to synchronize versions of the same variable used in different paths. For instance the fragment if $x>0$ then $x:=$ $x+10$ else $x:=x+20$ could be translated as $\left\{\right.$ if $x_{0}>0$ then $x_{1}:=$ $x_{0}+10$ else $\left.x_{2}:=x_{0}+20\right\} ; x_{3}:=\Phi\left(x_{1}, x_{2}\right)$. This means that the value assigned to $x_{3}$ depends on whether execution has reached this point through the first or the second branch of the conditional. In dynamic single-assignment form 27. variables may occur in multiple assignments in different paths.

Abstracting from the fact that assignments are replaced by assume statements, the original notion of passive form of [14] can be seen as a kind of dynamic SA where assignment instructions are replaced by assume commands, but similarly to the static notion, variable synchronization is achieved by introducing fresh variables assigned in both branches. Adapting this to standard imperative syntax, the above fragment would be translated as if $x_{0}>0$ then $x_{1}:=$ $x_{0}+10 ; x_{3}:=x_{1}$ else $x_{2}:=x_{0}+20 ; x_{3}:=x_{2}$. Our translation resembles the passify function introduced in [14, but there are significant differences: passify generates fresh variables abstractly, whereas we provide a concrete mechanism for this purpose; while passify only handles loop-free programs, our translation considers programs with loops annotated with invariants; passify is proved to be sound in the sense that it preserves the weakest precondition interpretation of programs, while our translation is proved to be sound with respect to the validity of Hoare triples, and moreover it is shown to be complete since it preserves derivability guided by the invariants. This is a crucial issue from the point of view of the completeness of using an intermediate SA form for verification.

Finally, passify does not generate version-optimal programs; the notion of version-optimal passive form, which uses the minimum number of version variables, is defined for unstructured programs in [16, together with a translation algorithm. In this form the above fragment becomes simply if $x_{0}>0$ then $x_{1}:=$ $x_{0}+10$ else $x_{1}:=x_{0}+20$. The algorithm differs from the translation of Appendix $\mathrm{A}$ in that it does not contemplate annotated loops, and no proof of soundness is given. However, they are similar in the use of variables: for loopfree programs, the DSA form produced by our translation is version-optimal.

Single-assignment forms have played an important role in two different families of efficient program verification techniques.

(I) In the generation of VCs using weakest precondition computations for programs based on Dijkstra's guarded commands [12. This setting is used as the basis for verification condition generation in ESC/Java and Boogie. For DSA programs, which appear here disguised as passive programs, weakest preconditions can be computed with quadratic size 1423 . Note that VCs are here created by assert commands instead of loop conditions. The approach was extended to programs with unstructured control flow $[5$ in an optimized way that produces linear-size VCs. It has also been shown that efficiently provable verification conditions can be generated using instead strongest postcondition computations [16].

There exists a single semantics of guarded commands programs, given by the definition of the predicate transformers, from which a VCGen is directly derived. This stands in contrast to our approach: soundness and relative com- 
proofs of soundness or completeness are available for bounded model checking of software.

\section{Conclusion}

Based on a Hoare-style logic for single-assignment programs, we have formalized a program verification technique that consists in translating annotated programs and specifications into an intermediate SA language, and generating VCs from these intermediate programs. An adaptation-complete variant of the logic is obtained by adding a dedicated consequence rule with a simple condition.

We have also shown how compact verification conditions can be produced directly from annotated SA programs. Assuming a right-associative representation of command sequences, the resulting VCs have linear size without requiring conversion of programs to unstructured form with goto commands as in [5].

Single-assignment intermediate forms are used extensively in software verification, both in model checking and in deductive verification; tools from both of these families eliminate iterating constructs before programs are converted to SA form. This stands in contrast with our work in this paper: we define rigorously a notion of single-assignment iterating program, and use it as the basis for a sound and complete verification technique, which includes the translation of annotated programs to SA form. We remark that the translation of loop invariants is a crucial component of the workflow, that doesn't trivially lead to completeness. To the best of our knowledge, this is the first time that completeness is established for a verification technique based on the use of an intermediate SA form for programs annotated with invariants.

Tools based on predicate transformers and bounded model checking incorporate many advanced features that our framework does not cover. For instance, Boogie includes automatic inference of loop invariants based on abstract interpretation, and CBMC, which natively uses a SAT (rather than SMT) solver, incorporates constant propagation and simplification functionality that is essential for making bounded verification work in practice. Still, our work here proposes a common theoretical foundation for program verification based on intermediate single-assignment form, unifying ideas from predicate transformers and program logic, while at the same time presenting adaptation-completeness as a natural property of the single-assignment setting.

In fact, a bounded notion of program verification as implemented in bounded model checking, which also relies on conversion to SA form, may also fit the same common foundation. The idea, which we will explore in future work, is to formalize this notion in a deductive setting, by obtaining a semantically justified bounded version of the VCGen of Section 4 .

Acknowledgments. This work is financed by the ERDF - European Regional Development Fund through the Operational Programme for Competitiveness and Internationalisation - COMPETE 2020 Programme, and by National Funds through the FCT - Fundação para a Ciência e a Tecnologia (Portuguese Foundation for Science 
and Technology) within project "POCI-01-0145-FEDER-006961". The first author is also sponsored by FCT grant SFRH/BD/52236/2013.

\section{A A Translation to SA Form}

We define a translation function that transforms an annotated program into SA form. We start by introducing some auxiliary definitions to deal with variable versions. Without loss of generality, we will assume that the universe of variables of the SA programs consists of two parts: the variable identifier and a version (a non-empty list of positive numbers). We let $\operatorname{Var}^{\mathrm{SA}}=\operatorname{Var} \times \mathbb{N}^{+}$be the set of SA variables, and we will write $x_{l}$ to denote $(x, l) \in \operatorname{Var}^{\mathrm{SA}}$. We write $\Sigma^{\mathrm{SA}}=$ $\operatorname{Var}^{\mathrm{SA}} \rightarrow D$ for the set of states, with $D$ being the interpretation domain.

Consider the version function $\mathcal{V}: \operatorname{Var} \rightarrow \mathbb{N}^{+}$. The function $\widehat{\mathcal{V}}: \operatorname{Var} \rightarrow \operatorname{Var}^{\mathrm{SA}}$ is such that $\widehat{\mathcal{V}}(x)=x_{\mathcal{V}(x)}$. $\widehat{\mathcal{V}}$ is lifted to Exp and Assert in the obvious way, renaming the variables according to $\mathcal{V}$. Let $s \in \Sigma$ and $\mathcal{V}: \operatorname{Var} \rightarrow \mathbb{N}^{+}$. We define $\mathcal{V}(s) \in \operatorname{Var}^{\mathrm{SA}} \rightarrow D$ as the partial function $[\widehat{\mathcal{V}}(x) \mapsto s(x) \mid x \in \mathbf{V a r}]$. Moreover, for $s^{\prime} \in \Sigma^{\mathrm{SA}}, s^{\prime} \oplus \mathcal{V}(s)$ denotes the overriding of $s^{\prime}$ by $\mathcal{V}(s)$.

The translation function $T_{\mathrm{sa}}$ is presented in Figure 7 . At the bottom we show the function that receives a triple and transforms it into the SA form. This function uses the auxiliary function shown on top (with the same name but different type) that receives the initial version of each variable identifier and the annotated program, and returns a pair with the final version of each variable identifier and the SA translated program. The definition of the latter function relies in turn on various auxiliary functions that deal with the version list and version functions, and also generate renaming commands. The functions are defined using Haskell-like syntax; we assume that the renaming sequences $\mathcal{I}$ and $\mathcal{U}$, defined in the case of while commands, follow some predefined order established over Var (any order will do).

We will now show that $T_{\mathrm{sa}}$ is indeed an SA translation. The full details of the proofs and a translation example can be found in 24. Firstly, we prove that the $T_{\mathrm{sa}}$ translation preserves the operational semantics of the original programs. Let us first consider some lemmas.

Lemma 9. Let $\mathcal{V} \in \operatorname{Var} \rightarrow \mathbb{N}^{+}, s \in \Sigma$ and $s^{\prime} \in \Sigma^{\mathrm{SA}}$. If $\forall x \in \operatorname{Var} . s(x)=$ $s^{\prime}(\widehat{\mathcal{V}}(x))$, then $s^{\prime}=s_{0}^{\prime} \oplus \mathcal{V}(s)$ for some $s_{0}^{\prime} \in \Sigma^{\mathrm{SA}}$.

Lemma 10. Let $e \in \mathbf{E x p}, \phi \in$ Assert, $\mathcal{V} \in \operatorname{Var} \rightarrow \mathbb{N}^{+}, s \in \Sigma$ and $s^{\prime} \in \Sigma^{\mathrm{SA}}$.

1. $\llbracket \widehat{\mathcal{V}}(e) \rrbracket\left(s^{\prime} \oplus \mathcal{V}(s)\right)=\llbracket e \rrbracket(s)$

2. $\llbracket \widehat{\mathcal{V}}(\phi) \rrbracket\left(s^{\prime} \oplus \mathcal{V}(s)\right)=\llbracket \phi \rrbracket(s)$

Lemma 11. Let $C \in \mathbf{A C o m m}$ and $\mathcal{V} \in \operatorname{Var} \rightarrow \mathbb{N}^{+}$. If $T_{\mathrm{sa}}(\mathcal{V}, C)=\left(\mathcal{V}^{\prime}, C^{\prime}\right)$, then for every $x \notin \operatorname{Asgn}(C), \mathcal{V}(x)=\mathcal{V}^{\prime}(x)$. 


$$
\begin{aligned}
& T_{\mathrm{sa}}:\left(\operatorname{Var} \rightarrow \mathbb{N}^{+}\right) \times \mathbf{A C o m m} \rightarrow\left(\operatorname{Var} \rightarrow \mathbb{N}^{+}\right) \times \mathbf{A C o m m}^{\mathrm{sA}} \\
& T_{\mathrm{sa}}(\mathcal{V}, \text { skip })=(\mathcal{V}, \text { skip }) \\
& T_{\mathrm{sa}}(\mathcal{V}, x:=e)=\left(\mathcal{V}[x \mapsto \operatorname{next}(\mathcal{V}(x))], x_{\operatorname{next}(\mathcal{V}(x))}:=\widehat{\mathcal{V}}(e)\right) \\
& T_{\mathrm{sa}}\left(\mathcal{V}, C_{1} ; C_{2}\right)=\left(\mathcal{V}^{\prime \prime}, C_{1}^{\prime} ; C_{2}^{\prime}\right) \\
& \text { where }\left(\mathcal{V}^{\prime}, C_{1}^{\prime}\right)=T_{\text {sa }}\left(\mathcal{V}, C_{1}\right) \\
& \left(\mathcal{V}^{\prime \prime}, C_{2}^{\prime}\right)=T_{\mathrm{sa}}\left(\mathcal{V}^{\prime}, C_{2}\right) \\
& \text { where }\left(\mathcal{V}^{\prime}, C_{t}^{\prime}\right)=T_{\mathrm{sa}}\left(\mathcal{V}, C_{t}\right) \\
& \left(\mathcal{V}^{\prime \prime}, C_{f}^{\prime}\right)=T_{\mathrm{sa}}\left(\mathcal{V}, C_{f}\right) \\
& \text { where } \mathcal{I} \quad=\left[x_{\operatorname{new}}(\mathcal{V}(x)):=x_{\mathcal{V}(x)} \mid x \in \operatorname{Asgn}(C)\right] \\
& \mathcal{V}^{\prime} \quad=\mathcal{V}[x \mapsto \operatorname{new}(\mathcal{V}(x)) \mid x \in \operatorname{Asgn}(C)] \\
& \left(\mathcal{V}^{\prime \prime}, C^{\prime}\right)=T_{\mathrm{sa}}\left(\mathcal{V}^{\prime}, C\right) \\
& \mathcal{U}=\left[x_{\operatorname{new}}(\mathcal{V}(x)):=x_{\mathcal{V}^{\prime \prime}(x)} \mid x \in \operatorname{Asgn}(C)\right] \\
& \mathcal{V}^{\prime \prime \prime} \quad=\mathcal{V}^{\prime \prime}\left[x \mapsto \operatorname{jump}(l) \mid x_{l} \in \operatorname{dom}(\mathcal{U})\right]
\end{aligned}
$$

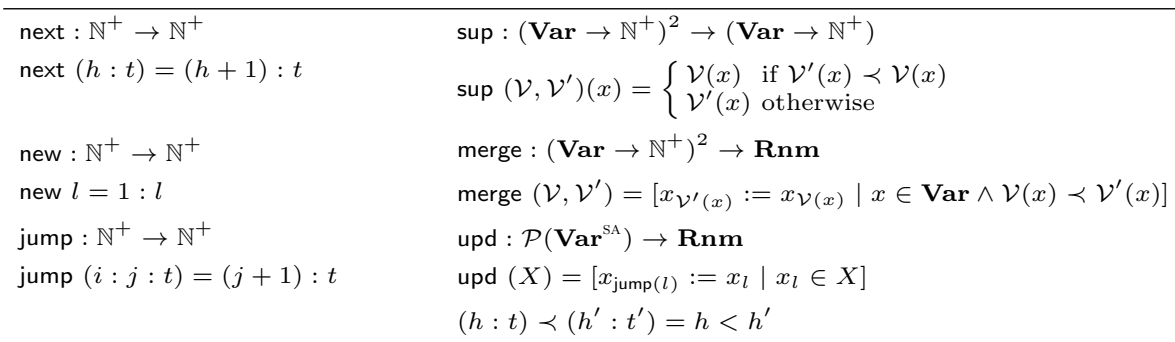

$T_{\mathrm{sa}}:$ Assert $\times$ AComm $\times$ Assert $\rightarrow$ Assert $^{\mathrm{SA}} \times \mathbf{A C o m m}^{\mathrm{SA}} \times$ Assert $^{\mathrm{SA}}$

$T_{\text {sa }}(\phi, C, \psi)=\left(\widehat{\mathcal{V}}(\phi), C^{\prime}, \widehat{\mathcal{V}^{\prime}}(\psi)\right), \quad$ where $\left(\mathcal{V}^{\prime}, C^{\prime}\right)=T_{\text {sa }}(\mathcal{V}, C)$, for some $\mathcal{V} \in \operatorname{Var} \rightarrow \mathbb{N}^{+}$

Fig. 7: SA translation function

Lemma 12. Let $C_{t} \in \mathbf{A C o m m}, \mathcal{V} \in \operatorname{Var} \rightarrow \mathbb{N}^{+}, s_{i}, s_{f} \in \Sigma, s^{\prime}, s_{f}^{\prime} \in \Sigma^{\mathrm{SA}}$, $\mathcal{V}^{\prime}=\mathcal{V}\left[x \mapsto \operatorname{new}(\mathcal{V}(x)) \mid x \in \operatorname{Asgn}\left(C_{t}\right)\right], T_{\mathrm{sa}}\left(\mathcal{V}^{\prime}, C_{t}\right)=\left(\mathcal{V}^{\prime \prime}, C_{t}^{\prime}\right)$, $\mathcal{U}=\left[x_{\text {new }(\mathcal{V}(x))}:=x_{\mathcal{V}^{\prime \prime}(x)} \mid x \in \operatorname{Asgn}\left(C_{t}\right)\right]$. If $\left\langle\right.$ while $b$ do $\left.\left\lfloor C_{t}\right\rfloor, s_{i}\right\rangle \sim s_{f}$ and $\left\langle\right.$ while $\mathcal{V}^{\prime}(b)$ do $\left.\left\{\left\lfloor\mathcal{W}\left(C_{t}^{\prime}\right)\right\rfloor ; \mathcal{U}\right\} ; \operatorname{upd}(\operatorname{dom}(\mathcal{U})), s^{\prime} \oplus \mathcal{V}\left(s_{i}\right)\right\rangle \sim s_{f}^{\prime}$, then $\forall x \in \operatorname{Var} . s_{f}(x)=s_{f}^{\prime}\left(\widehat{\mathcal{V}}^{\prime}(x)\right)$.

Proposition 11. Let $C \in \mathbf{A C o m m}, \mathcal{V} \in \operatorname{Var} \rightarrow \mathbb{N}^{+}, s_{i}, s_{f} \in \Sigma, s^{\prime}, s_{f}^{\prime} \in \Sigma^{\mathrm{SA}}$ and $T_{\mathrm{sa}}(\mathcal{V}, C)=\left(\mathcal{V}^{\prime}, C^{\prime}\right)$. If $\left\langle\lfloor C\rfloor, s_{i}\right\rangle \sim s_{f}$ and $\left\langle\left\lfloor\mathcal{W}\left(C^{\prime}\right)\right\rfloor, s^{\prime} \oplus \mathcal{V}\left(s_{i}\right)\right\rangle \sim s_{f}^{\prime}$, then $\forall x \in \operatorname{Var} . s_{f}(x)=s_{f}^{\prime}\left(\widehat{\mathcal{V}}^{\prime}(x)\right)$.

Proof. By induction on the structure of $C$. 
Secondly, we prove that lifting the translation function to Hoare triples is sound, i.e., if the translated triple is valid then the original triple is also valid.

Lemma 13. Let $C \in \mathbf{A C o m m}, \mathcal{V} \in \mathbf{V a r} \rightarrow \mathbb{N}^{+}$, and $s_{i}, s_{f} \in \Sigma$. If $\left\langle\lfloor C\rfloor, s_{i}\right\rangle \leadsto$ $s_{f}$ and $T_{\mathrm{sa}}(\mathcal{V}, C)=\left(\mathcal{V}^{\prime}, C^{\prime}\right)$, then $\left\langle\left\lfloor\mathcal{W}\left(C^{\prime}\right)\right\rfloor, s_{1}^{\prime} \oplus \mathcal{V}\left(s_{i}\right)\right\rangle \sim s_{2}^{\prime} \oplus \mathcal{V}^{\prime}\left(s_{f}\right)$, for some $s_{1}^{\prime}, s_{2}^{\prime} \in \Sigma^{\mathrm{SA}}$.

Proof. By induction on the structure of $C$ using Proposition 11.

Proposition 12. Let $C \in \mathbf{A C o m m}, \phi, \psi \in$ Assert, $\mathcal{V} \in$ Var $\rightarrow \mathbb{N}^{+}$and $T_{\mathrm{sa}}(\mathcal{V}, C)=\left(\mathcal{V}^{\prime}, C^{\prime}\right)$.

If $\models\{\widehat{\mathcal{V}}(\phi)\}\left\lfloor\mathcal{W}\left(C^{\prime}\right)\right\rfloor\left\{\widehat{\mathcal{V}}^{\prime}(\psi)\right\}$, then $\models\{\phi\}\lfloor C\rfloor\{\psi\}$.

Proof. Follows from Lemmas 13 and 10 and Propositon 11 .

Finally, we will show that $\mathrm{Hg}$-derivability is preserved, i.e. if a Hoare triple for an annotated program is derivable in $\mathrm{Hg}$, then the translated triple is also derivable in $\mathrm{Hg}$. Again we start by stating some lemmas.

Lemma 14. Let $\mathcal{V}, \mathcal{V}^{\prime} \in \operatorname{Var} \rightarrow \mathbb{N}^{+}$and $\psi \in$ Assert. The following hold:

1. $\left.\vdash_{\mathrm{Hg}}\{\widehat{\mathcal{V}}(\psi)\} \operatorname{merge}\left(\mathcal{V}, \mathcal{V}^{\prime}\right)\left\{\widehat{\sup \left(\mathcal{V}, \mathcal{V}^{\prime}\right.}\right)(\psi)\right\}$

2. $\vdash_{\text {Hg }}\{\widehat{\mathcal{V}}(\psi)\} \operatorname{merge}\left(\mathcal{V}^{\prime}, \mathcal{V}\right)\left\{\sup \left(\mathcal{V}, \mathcal{V}^{\prime}\right)(\psi)\right\}$

Lemma 15. Let $\mathcal{I} \in \mathbf{R n m}$ and $\phi \in$ Assert. Then $\vdash_{\mathrm{Hg}}\{\phi\} \mathcal{I}\left\{\mathcal{I}^{-1}(\phi)\right\}$ holds.

Lemma 16. Let $\mathcal{V} \in \operatorname{Var} \rightarrow \mathbb{N}^{+}, C \in \mathbf{A C o m m}, \mathcal{V}^{\prime}=\mathcal{V}[x \mapsto \operatorname{new}(\mathcal{V}(x)) \mid x \in$ $\operatorname{Asgn}(C)], T_{\mathrm{sa}}\left(\mathcal{V}^{\prime}, C\right)=\left(\mathcal{V}^{\prime \prime}, C^{\prime}\right)$ and $\mathcal{U}=\left[x_{\operatorname{new}(\mathcal{V}(x))}:=x_{\mathcal{V}^{\prime \prime}(x)} \mid x \in \operatorname{Asgn}(C)\right]$. The derivation $\vdash_{\mathrm{Hg}}\left\{\widehat{\mathcal{V}^{\prime \prime}}(\theta)\right\} \mathcal{U}\left\{\widehat{\mathcal{V}}^{\prime}(\theta)\right\}$ holds.

Lemma 17. Let $\mathcal{V} \in \operatorname{Var} \rightarrow \mathbb{N}^{+}, C \in \mathbf{A C o m m}, \mathcal{V}^{\prime}=\mathcal{V}[x \mapsto \operatorname{new}(\mathcal{V}(x))$ $x \in \operatorname{Asgn}(C)], T_{\text {sa }}\left(\mathcal{V}^{\prime}, C\right)=\left(\mathcal{V}^{\prime \prime}, C^{\prime}\right), \mathcal{U}=\left[x_{\text {new }(\mathcal{V}(x))}:=x_{\mathcal{V}^{\prime \prime}(x)} \mid x \in \operatorname{Asgn}(C)\right]$ and $\mathcal{V}^{\prime \prime \prime}=\mathcal{V}^{\prime \prime}\left[x \mapsto \operatorname{jump}(l) \mid x_{l} \in \operatorname{dom}(\mathcal{U})\right]$. The following derivation holds $\vdash_{\text {Hg }}\left\{\widehat{\mathcal{V}}^{\prime}(\psi)\right\} \operatorname{upd}(\operatorname{dom}(\mathcal{U}))\left\{\widehat{\mathcal{V}^{\prime \prime \prime}}(\psi)\right\}$.

Proposition 13. Let $C \in$ AComm, $\phi, \psi \in$ Assert, $\mathcal{V} \in$ Var $\rightarrow \mathbb{N}^{+}$and $T_{\mathrm{sa}}(\mathcal{V}, C)=\left(\mathcal{V}^{\prime}, C^{\prime}\right)$. If $\vdash_{\mathrm{Hg}}\{\phi\} C\{\psi\}$, then $\vdash_{\mathrm{Hg}}\{\widehat{\mathcal{V}}(\phi)\} \mathcal{W}\left(C^{\prime}\right)\left\{\widehat{\mathcal{V}}^{\prime}(\psi)\right\}$.

Proof. By induction on the structure of $\vdash_{\mathrm{Hg}}\{\phi\} C\{\psi\}$.

It is now immediate that $T_{\mathrm{sa}}$ conforms to Definition 6 .

Proposition 14. The $T_{\mathrm{sa}}$ function of Figure 7 is an $S A$ translation.

Proof. Follows directly from Propositions 12 and 13 


\section{References}

1. Pierre America and Frank de Boer. Proving total correctness of recursive procedures. Information and Computation, 84(2):129-162, 1990.

2. Krzysztof R. Apt. Ten years of Hoare's logic: A survey - part 1. ACM Transactions on Programming Languages and Systems, 3(4):431-483, 1981.

3. John Barnes. High integrity software: The SPARK approach to safety and security. Addison-Wesley, 2003.

4. Michael Barnett, Bor-Yuh Evan Chang, Robert DeLine, Bart Jacobs, and K. Rustan M. Leino. Boogie: A modular reusable verifier for object-oriented programs. In Proceedings of FMCO 2005, volume 4111 of Lecture Notes in Computer Science, pages 364-387. Springer, 2005.

5. Michael Barnett and K. Rustan M. Leino. Weakest-precondition of unstructured programs. ACM SIGSOFT Software Engineering Notes, 31(1):82-87, 2006.

6. Mike Barnett, K. Rustan, M. Leino, and Wolfram Schulte. The Spec\# programming system: An overview. In Proceedings of CASSIS 2004, volume 3362 of Lecture Notes in Computer Science, pages 49-69. Springer, 2004.

7. Patrick Baudin, Pascal Cuoq, Jean-Christophe Filliâtre, Claude Marché, Benjamin Monate, Yannick Moy, and Virgile Prevosto. ACSL: ANSI/ISO C Specification Language. CEA LIST and INRIA, 2010.

8. Edmund Clarke, Daniel Kroening, and Flavio Lerda. A tool for checking ANSI-C programs. In Proceedings of TACAS 2004, pages 168-176. Springer, 2004.

9. Stephen A. Cook. Soundness and completeness of an axiom system for program verification. SIAM Journal on Computing, 7(1):70-90, 1978.

10. Loc Correnson, Pascal Cuoq, Armand Puccetti, and Julien Signoles. Frama-C user manual. Available from the Frama-C website, http://frama-c.com, 2010.

11. Ron Cytron, Jeanne Ferrante, BK Rosen, Mark N Wegman, and F. K. Zadeck. Efficiently computing static single assignment form and the control dependence graph. ACM Transactions on Programming Languages and Systems, 13(4):451490, October 1991.

12. Edsger W. Dijkstra and Carel S. Scholten. Predicate calculus and program semantics. Springer, 1990.

13. Jean-Christophe Filliâtre and Andrei Paskevich. Why3 - where programs meet provers. In Proceedings of ESOP 2013, volume 7792 of Lecture Notes in Computer Science, pages 125-128. Springer, 2013.

14. Cormac Flanagan and James B. Saxe. Avoiding exponential explosion: generating compact verification conditions. In Proceedings of POPL 2001, pages 193-205. ACM 2001.

15. Mike Gordon and Hélène Collavizza. Forward with Hoare. In Reflections on the Work of C.A.R. Hoare, History of Computing, pages 101-121. Springer, 2010.

16. Radu Grigore, Julien Charles, Fintan Fairmichael, and Joseph Kiniry. Strongest postcondition of unstructured programs. In Proceedings of FTfJP 2009, pages 6:1-6:7. ACM, 2009.

17. C. A. R. Hoare. An axiomatic basis for computer programming. Communications of the ACM, 12(10):576-580, 1969.

18. C. A. R. Hoare. Procedures and parameters: An axiomatic approach. In Proceeedings of Symposium on Semantics of Algorithmic Languages, volume 188 of Lecture Notes in Mathematics. Springer, 1971.

19. Ranjit Jhala and Rupak Majumdar. Software model checking. ACM Computing Surveys, 41(4):21, October 2009. 
20. Thomas Kleymann. Hoare logic and auxiliary variables. Formal Aspects of Computing, 11(5):541-566, 1999.

21. Daniel Kroening. Software verification. In Handbook of Satisfiability, pages 505532. IOS Press, 2009.

22. K. Rustan M. Leino, James B. Saxe, and Raymie Stata. Checking Java programs via guarded commands. In Proceedings of ECOOP 1999, pages 110-111. Springer, 1999.

23. K. Rustan M. Leino. Efficient weakest preconditions. Information Processing Letters, 93(6):281-288, 2005.

24. Cláudio B. Lourenço, Maria J. Frade and Jorge S. Pinto. A SingleAssignment Translation for Annotated Programs. ArXiv e-prints, available from http://arxiv.org/abs/1601.00584, 2016.

25. Claude Marché, Christine Paulin-Mohring, and Xavier Urbain. The KRAKATOA tool for certification of JAVA/JAVACARD programs annotated in JML. The Journal of Logic and Algebraic Programming, 58(1-2):89-106, 2004.

26. John C. Reynolds. Separation logic: A logic for shared mutable data structures. In LICS 2002, pages 55-74. IEEE Computer Society, 2002.

27. Peter Vanbroekhoven, Gerda Janssens, Maurice Bruynooghe, and Francky Catthoor. A practical dynamic single assignment transformation. ACM Transactions on Design Automation of Electronic Systems, 12(4):40, 2007. 